\title{
Recovery of Bulk Proton Magnetization and Sensitivity Enhancement in Ultrafast Magic-Angle Spinning Solid-State NMR
}

\author{
Jean-Philippe Demers, ${ }^{* \dagger}$ Vinesh Vijayan, ${ }^{\ddagger}$ and Adam Lange ${ }^{\dagger}$ \\ Department of NMR-Based Structural Biology, Max Planck Institute for Biophysical Chemistry, 37077 Göttingen, Germany
}

Supporting Information

ABSTRACT: The sensitivity of solid-state NMR experiments is limited by the proton magnetization recovery delay and by the duty cycle of the instrument. Ultrafast magic-angle spinning (MAS) can improve the duty cycle by employing experiments with low-power radio frequency (RF) irradiation which reduce RF heating. On the other hand, schemes to reduce the magnetization recovery delay have been proposed for low MAS rates, but the enhancements rely on selective transfers where the bulk of the ${ }^{1} \mathrm{H}$ magnetization pool does not

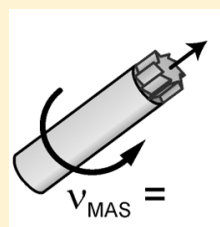

$65 \mathrm{kHz}$

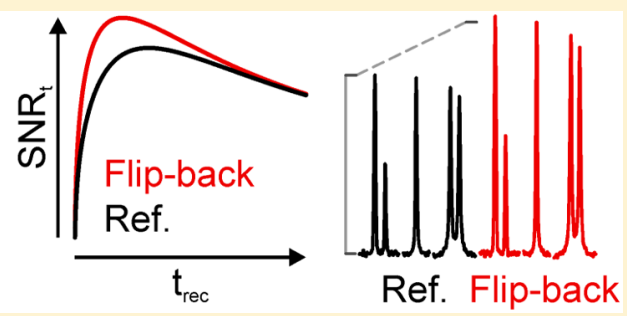
contribute to the transfer. We demonstrate here that significant sensitivity enhancements for selective and broadband experiments are obtained at ultrafast MAS by preservation and recovery of bulk ${ }^{1} \mathrm{H}$ magnetization. We used $\left[{ }^{13} \mathrm{C}\right.$, $\left.{ }^{15} \mathrm{~N}\right]$-labeled glutamine as a model compound, spinning in a $1.3 \mathrm{~mm}$ rotor at a MAS frequency of $65 \mathrm{kHz}$. Using low-power ${ }^{1} \mathrm{H} \mathrm{RF}(13.4 \mathrm{kHz})$, we obtain efficient ${ }^{1} \mathrm{H}$ spin locking and ${ }^{1} \mathrm{H}-{ }^{13} \mathrm{C}$ decoupling at ultrafast MAS. As a result, large amounts of ${ }^{1} \mathrm{H}$ magnetization, from $35 \%$ to $42 \%$ of the initial polarization, are preserved after cross-polarization and decoupling. Restoring this magnetization to the longitudinal axis using a flip-back pulse leads to an enhancement of the sensitivity, an increase ranging from $14 \%$ to $21 \%$ in the maximal achievable sensitivity regime and from $24 \%$ to $50 \%$ in the fast pulsing regime, and to a shortening of the optimal recycling delay to $68 \%$ of its original duration. The analysis of the recovery and sensitivity curves reveals that the sensitivity gains do not rely on a selective transfer where few protons contribute but rather on careful conservation of bulk ${ }^{1} \mathrm{H}$ magnetization. This makes our method compatible with broadband experiments and uniformly labeled materials, in contrast to the enhancement schemes proposed for low MAS. We tested seven different cross-polarization schemes and determined that recovery of bulk ${ }^{1} \mathrm{H}$ magnetization is a general method for sensitivity enhancement. The physical insight gained about the behavior of proton magnetization sharing under spin lock will be helpful to break further sensitivity boundaries, when even higher external magnetic fields and faster spinning rates are employed.

\section{INTRODUCTION}

Ultrafast magic-angle spinning (MAS) offers several attractive advantages over MAS experiments at slower spinning rates. ${ }^{1-3}$ Such advantages include the use of low-power pulse elements, the reduction of radio frequency (RF) heating, and efficient averaging of the anisotropic part of nuclear interactions such as chemical shift anisotropies, dipolar couplings, and quadrupolar couplings. As a consequence, several new applications have been enabled by the use of ultrafast spinning: study of quantity-limited samples, study of proteins containing paramagnetic centers, ${ }^{4-7}$ measurement of dynamic processes, ${ }^{8,9}$ measurement of $\mathrm{X}-\mathrm{H}$ distances, ${ }^{10}$ improved coherence lifetimes during through-bond correlation experiments, ${ }^{11-13}$ and new symmetry-based recoupling sequences. ${ }^{14-17}$

At fast MAS $(20-40 \mathrm{kHz})$ and ultrafast MAS $(>40 \mathrm{kHz})$, however, the signal-to-noise ratio is compromised due to the reduction of the rotor dimensions and decrease of the NMRactive sample volume. Different strategies have been implemented in order to restore a high sensitivity at fast and ultrafast MAS: paramagnetic doping aiming at acceleration of longitudinal ${ }^{1} \mathrm{H}$ relaxation combined with the use of short recycling delays, ${ }^{18-22}$ proton-detected experiments on protonated materials, ${ }^{23-25}$ and deuterated samples; ${ }^{7,26-32}$ on the other hand, ultrafast MAS probe heads integrated into a dynamic nuclear polarization $(\mathrm{DNP})^{33,34}$ setup are being actively developed.

Another important concept employed to boost NMR sensitivity stems from the realization that a large portion of magnetization remains unused after an experiment and gives rise to two different strategies for sensitivity enhancement. In the first strategy, the magnetization remaining on low- $\gamma$ nuclei (e.g., ${ }^{13} \mathrm{C}$ ) is utilized. One method for sensitivity enhancement of solid-state NMR named RELOAD-CP was proposed, where the bulk ${ }^{13} \mathrm{C}$ polarization is stored along the $+z$ axis and used to accelerate the recovery of selectively excited ${ }^{13} \mathrm{C}$ nuclei. ${ }^{35}$ In another method, the "afterglow" signal remaining on ${ }^{15} \mathrm{~N}$ after a first band-selective double cross-polarization experiment to $\mathrm{C}_{\alpha}$ is transferred to the $C^{\prime}$ carbons for an immediate second detection. ${ }^{36,37}$ Similarly, parallel acquisition setups have been developed ${ }^{38}$ as well as experiments with multiple acquisition periods-simultaneous cross-polarization (CP) to ${ }^{15} \mathrm{~N}$ and ${ }^{13} \mathrm{C}$ in $\mathrm{DUMAS}^{39,40}$ and

Received: December 1, 2014

Revised: January 13, 2015

Published: January 14, 2015 
MEIOSIS ${ }^{41}$ - or time-shared acquisition with simultaneous acquisition of ${ }^{15} \mathrm{~N}-{ }^{13} \mathrm{C}$ and ${ }^{13} \mathrm{C}-{ }^{13} \mathrm{C}$ correlations in TSTSAR ${ }^{42}$

In the second strategy, the magnetization remaining on protons is recovered and used to accelerate the apparent magnetization recovery rate after an experiment, allowing the shortening of the recycling delay. This strategy is exploited in solution-state $\mathrm{NMR}^{43}$ for example in the LTROSY, ${ }^{44}$ SOFAST, $^{45}$ and $\operatorname{COST}^{46}$ families of experiments. In 1978, Tegenfeldt and Haeberlen first proposed to employ this strategy in solid-state NMR for experiments directly detected on a low- $\gamma$ nucleus, by applying a flip-back pulse on the ${ }^{1} \mathrm{H}$ channel after cross-polarization and decoupling. ${ }^{47}$ A special case where this strategy is particularly efficient is when only a few protons contribute to the polarization transfer, for instance when carbons are selectively excited ${ }^{48}$ or in the case of natural abundance ${ }^{13} \mathrm{C}$ materials. ${ }^{49}$ The flip-back pulse was also employed in applications to pharmaceuticals ${ }^{50}$ and deuterated proteins. ${ }^{51,52}$

The magnetization recovery strategy suffers however from two limitations in the low MAS $(<20 \mathrm{kHz})$ and fast MAS $(20-40 \mathrm{kHz})$ regimes: (i) the pulse sequence elements employed on the ${ }^{1} \mathrm{H}$ channel need to preserve a sufficient amount of polarization; (ii) these pulse elements must be compatible with efficient magnetization transfer and heteronuclear decoupling schemes. Following our previous studies on spin-locked ${ }^{1} \mathrm{H}$ magnetization, ${ }^{53,54}$ we demonstrate in the current study that favorable conditions are obtained at ultrafast MAS. The conditions employed provide a general method for sensitivity enhancement which can be combined with a large variety of $\mathrm{CP}$ schemes and which does not require selective carbon excitation, making the recovery enhancement strategy suitable for the recording of broadband excitation spectra on uniformly labeled and natural abundance samples altogether. This method can be combined with the second-order crosspolarization (SOCP) scheme $\mathrm{e}^{53}$ and its broadband variation, the amplitude-modulated SOCP scheme (MOD-CP). ${ }^{54}$ Those two schemes were shown to offer a sizable enhancement-the sensitivity is increased by $\sim 50 \%$ in reference to hard-power CP schemes ${ }^{53,54}$ — and only require a low RF power.

\section{EXPERIMENTAL METHODS}

Sample Preparation and Solid-State NMR Spectroscopy. A $1.3 \mathrm{~mm}$ rotor was packed with $2.91 \mathrm{mg}$ of uniformly $\left[{ }^{13} \mathrm{C},{ }^{15} \mathrm{~N}\right]$ labeled L-glutamine (Cambridge Isotope Laboratories, Cambridge, MA, USA). All spectra were recorded on a standard-bore 18.8 $\mathrm{T}$ instrument ( $800 \mathrm{MHz}{ }^{1} \mathrm{H}$ Larmor frequency) equipped with a $1.3 \mathrm{~mm}$ DVT triple-resonance probe head (Bruker BioSpin, Rheinstetten, Germany) and running the TopSpin 2.1 acquisition software. The source code for pulse programs and composite pulse decoupling programs presented in Figure 2 is provided in the Supporting Information. The full phase cycles, description of the parameters, and instructions for using the programs on TopSpin 2.x and 3.x systems are included. A MAS rate of $65.0 \mathrm{kHz}$ was employed. A sample temperature of $40{ }^{\circ} \mathrm{C}$ was obtained by regulating the temperature of the cooling nitrogen gas at $-25^{\circ} \mathrm{C}$ with a gas flow in the range of $935-1200 \mathrm{~L} / \mathrm{h}$ using a BCU-X accessory.

In order to calibrate the RF nutation frequencies $\left(\nu_{1}^{1} \mathrm{H}\right.$ for the proton channel, $\nu_{1}^{13} \mathrm{C}$ for the carbon channel), we recorded nutation experiments at a series of amplifier attenuation values $(\mathrm{dB})$. In the nutation curve, the average difference between two zero-crossing points was used to determine the length of the $180^{\circ}$ pulse at a particular attenuation value. The RF nutation frequencies produced by the amplifiers were linear over the full range of used attenuation values $\left(R^{2}=0.999\right)$, and RF nutation frequencies were calculated by linear regression from the calibrated values.

Extraction of Signal Intensities. A baseline correction is first applied on the individual frequency-domain spectra by fitting areas devoid of signal with a piecewise polynomial spline function ${ }^{55}$ (six pieces; polynomial order, 4). The spectral intensity as a function of frequency, $I_{(\nu)}($ eq 1), results from the addition of the resonance spectra $I_{(\nu)}$ contributed from each individual nucleus.

$$
I_{(\nu)}=\sum_{j=1}^{N} I_{(\nu)}^{j}
$$

We describe $P_{(\nu)}$ with a mixed Lorentzian/Gaussian function (Voigt profile), which is the convolution $(*)$ of a Lorentzian line-shape $L_{(\nu)}$ (eq 2) with a Gaussian line-shape, $G_{(\nu)}$ (eq 3 ). The Lorentzian line shape is associated with the apparent transverse dephasing rate of a resonance peak while the Gaussian line shape is associated with the heterogeneous broadening of a resonance peak due to inhomogeneity of the sample and static field. Parameters for those functions are the isotropic chemical shift, $\nu_{0}(\mathrm{ppm})$; a Gaussian line-width parameter, $\sigma$; and a Lorentz line-width parameter, $\Gamma$.

$$
\begin{aligned}
L_{(\nu)} & =\frac{\Gamma}{2 \pi\left(\left(\nu-\nu_{0}\right)^{2}+\left(\frac{1}{2} \Gamma\right)^{2}\right)} \\
G_{(\nu)} & =\frac{1}{\sigma \sqrt{2 \pi}} \mathrm{e}^{-\nu^{2} / 2 \sigma^{2}}
\end{aligned}
$$

The Voigt profile (eq 4) has four parameters: $\nu_{0}, \sigma, \Gamma$, and the peak volume, $a_{0}$ (arbitrary unit). The mixed function was computed from the real part of the Faddeeva function (eq 5), where erfc is the complex complementary error function. ${ }^{56}$

$$
\begin{aligned}
& I_{(\nu)}{ }^{j}=a_{0} L_{(\nu)}{ }^{*} G_{(\nu)} \\
& I_{(\nu)}{ }^{j}=a_{0} \operatorname{Re}\left[w_{\left(\left(\nu-\nu_{0}+(1 / 2) \Gamma\right) / \sigma \sqrt{2}\right)}\right], \quad w_{(z)}=\mathrm{e}^{-z^{2}} \operatorname{erfc}(-i z)
\end{aligned}
$$

The full width at half-height (fwhh) for the Lorentzian, Gaussian, and Voigt functions are given by ${ }^{57}$

$$
\begin{aligned}
& f_{\mathrm{L}}=\Gamma \\
& f_{\mathrm{G}}=\sigma \sqrt{2 \ln 2} \\
& f_{\mathrm{V}} \approx 0.5346 f_{\mathrm{L}}+\sqrt{0.2166 f_{\mathrm{L}}^{2}+f_{\mathrm{G}}^{2}}
\end{aligned}
$$

For a free induction decay (FID) recorded in a given number of scans, $n_{\text {scan }}$ the total signal energy $S_{\text {tot }}$ is simply the sum of individual peak volumes, $a_{0}$, as both $L_{(\nu)}$ and $G_{(\nu)}$ have a constant unit volume:

$$
S_{\text {tot }}=\sum_{j=1}^{N} a_{0}^{j}
$$

The peak volumes for the five ${ }^{13} \mathrm{C}$ resonances of L-glutamine are obtained by least-squares fitting of the spectrum with five individual mixed functions $I_{(\nu)}$. An initial parameter estimate is obtained from the piecewise fitting of spectral regions to a single 
mixed function; the final fitting optimizes all five mixed functions simultaneously. For Figures 1a, 3, 4, and 5, the parameters $\nu_{0}, \sigma$, and $\Gamma$ are optimized on a reference spectrum (sum of all spectra) and then kept fixed while $a_{0}$ values are further optimized for each spectrum of a series. For Figure $1 b, \nu_{0}$ is determined from the reference spectrum, values for $\sigma$ and $a_{0}$ are determined globally for all spectra and successively fixed, and then values for $\Gamma$ are optimized for each individual spectrum.

Measurement of Remaining ${ }^{1} \mathrm{H}$ Magnetization Level and ${ }^{1} \mathrm{H}$ Magnetization Recovery Curves. During an arbitrary pulse sequence, the initial ${ }^{1} \mathrm{H}$ magnetization $M_{0}^{z}$ reduces to a final level $M_{\mathrm{f}}^{z}$ (eq 8). The fractions of preserved ${ }^{1} \mathrm{H}$ magnetization, $f_{0}$, were determined, using the pulse sequence in Figure $2 \mathrm{~d}$, for the following pulse elements: (i) a ${ }^{1} \mathrm{H}$ spin lock, (ii) crosspolarization, and (iii) cross-polarization followed by continuouswave (cw) decoupling. After excitation by a $90^{\circ}$ pulse on ${ }^{1} \mathrm{H}$, a "mock" experiment was carried out with the aforementioned pulse elements; the remaining ${ }^{1} \mathrm{H}$ magnetization amount was then read immediately through cross-polarization to ${ }^{13} \mathrm{C}\left(t_{\mathrm{rec}}=0\right)$. The CP conditions were kept identical for the "mock" and for the "read" experiment, such that the reported levels originate solely from the pool of protons that contribute to cross-polarization. Any ${ }^{13} \mathrm{C}$ magnetization already present after the mock experiment was filtered out by phase cycling of the aforementioned pulse elements. The full phase cycles are presented in the Supporting Information. To ensure equal amounts of initial ${ }^{1} \mathrm{H}$ magnetization, each repetition of the experiment was preceded by two $6 \mathrm{~ms}$ purge pulses at $\nu_{1}^{1} \mathrm{H}=83 \mathrm{kHz}$ with phase $x,-x$, followed by a recycling delay of $10 \mathrm{~s}$. For $\mathrm{i}$ and ii, the ${ }^{1} \mathrm{H}$ spin-lock pulse and cross-polarization ${ }^{1} \mathrm{H}$ pulse have the same duration and power level, as listed in Table 1). For iii, the $\mathrm{cw}$ decoupling was applied for $8 \mathrm{~ms}$ with a RF frequency $\nu_{1}^{1} \mathrm{H}=13.4 \mathrm{kHz}$. The crosspolarization ${ }^{1} \mathrm{H}$ pulse and the $\mathrm{cw}$ decoupling pulse are always applied along the same phase.

$$
M_{\mathrm{f}}^{z}=f_{0} M_{0}^{z}
$$

Magnetization recovery curves (Figure 4a) were recorded in a similar fashion (Figure 2d), with cross-polarization followed by $\mathrm{cw}$ decoupling in the mock experiment, with the addition that a variable recovery delay $t_{\text {rec }}=\tau$ was inserted prior to the read experiment. A recycling delay of $21.5 \mathrm{~s}$ was employed. Three series of experiments were recorded: (i) no pulse is applied, leading to rapid decay of transverse ${ }^{1} \mathrm{H}$ magnetization; (ii) a $90^{\circ}$ ${ }^{1} \mathrm{H}$ pulse is applied directly after the mock experiment to return magnetization to $+\hat{I}_{z}$ (flip-back); or (iii) a $90^{\circ}{ }^{1} \mathrm{H}$ pulse placed the magnetization along the $-\hat{I}_{z}$ axis (flip-down). Depending on the series of experiment, the following value for $M_{\mathrm{f}}^{z}$ is employed: (i) $M_{\mathrm{f}}^{z}=0$, (ii) $M_{\mathrm{f}}^{z}=f_{0} M_{0}^{z}$, or (iii) $M_{\mathrm{f}}^{z}=-f_{0} M_{0}^{z}$.

Assuming a monoexponential recovery behavior, the magnetization available for the read experiment is described by eq 9 . Alternatively, a more complex recovery behavior can be better described by a weighted sum of $N$ exponentials, each exponential with individual population, $p_{i}$; apparent relaxation time, $T_{1, i}$; and remaining fraction, $f_{0, i}$ (eq 10$)$.

$$
\begin{aligned}
& M_{(\tau)}^{z}=M_{\mathrm{eq}}-\left(M_{\mathrm{eq}}-M_{\mathrm{f}}^{z}\right) \exp \left(-\tau / T_{1, \mathrm{app}}^{1} \mathrm{H}\right. \\
& M_{(\tau)}^{z}=M_{\mathrm{eq}}-\sum_{i=1}^{N}\left[p_{i}\left(M_{\mathrm{eq}}-M_{\mathrm{f}, i}^{z}\right) \exp \left(-\tau / T_{1, i}\right)\right] / \sum_{i=1}^{N} p_{i}
\end{aligned}
$$

Measurement of Sensitivity in the Steady State. The sensitivity of a multiscan experiment as a function of $t_{\mathrm{rec}}=\tau$ was measured through cross-polarization. Experiments were recorded in three different conditions using the pulse sequence in Figure 2c: (i) no pulse applied after decoupling, (ii) with flip-back pulse, or (iii) with flip-down pulse. The sensitivity ${ }^{58}$ is described in the frequency domain by the signal-to-noise ratio per unit square root time $(\mathrm{S} / \mathrm{N})_{t}$ [eq 11]. The signal amplitude extracted from experiment is taken as the total signal energy $S_{\text {tot }}$ as described in Extraction of Signal Intensities.

$$
(\mathrm{S} / \mathrm{N})_{t}=\frac{S_{\mathrm{tot}}}{\sigma_{\text {noise }} \sqrt{t_{\text {tot }}}}
$$

The noise amplitude, $\sigma_{\text {noise }}$ as estimated from the mean standard deviation of independent regions of the baseline, was found to increase with the square root of $n_{\text {scan }}$ as in eq 12, where $k_{\text {noise }}$ is a proportionality constant. The number of scans of each experiment was adjusted to obtain a total experimental time, $t_{\text {tot }}$ of at least $200 \mathrm{~s} /$ replicate. A sufficient number of dummy scans were employed prior to the first recorded iteration, $n_{\text {dummy }} \geq$ $\max \left(0.08 n_{\text {scan }}, 2\right)$, such that the measured magnetization did not deviate by more than $2 \%$ from ideal steady state.

$$
\sigma_{\text {noise }}=k_{\text {noise }} \sqrt{n_{\text {scan }}}
$$

The sensitivity can be modeled as a function of the interscan delay for the three series of experiments, since the amount of ${ }^{13} \mathrm{C}$ magnetization detected through cross-polarization is directly related to the amount of ${ }^{1} \mathrm{H}$ magnetization at the beginning of the experiment $M_{0}^{z}$; the calculated signal energy $S_{\text {calc }}$ being related to $M_{0}^{z}$ by a proportionally constant:

$$
S_{\text {calc }}=\eta M_{0}^{z}
$$

The amount $M_{0}^{z}$ decreases with every scan in the initial regime, but approaches a steady-state value $M_{0, \text { SS }}^{z}$ after a large number of scans. The value of $M_{0, \text { SS }}^{z}$ can be found for arbitrary values of $f_{0}$ and $\mathrm{T}_{1, \text { app }}^{1} \mathrm{H}$ through a geometrical series ${ }^{43,59}$ assuming a monoexponential behavior for the recovery (eq 14) or for a multiexponential behavior (eq 15).

$$
\begin{aligned}
& M_{0, \mathrm{SS}(\tau)}^{z}=M_{\mathrm{eq}} \frac{1-\exp \left(-\tau / T_{1, \mathrm{app}}{ }^{1} \mathrm{H}\right)}{1-f_{0} \exp \left(-\tau / T_{1, \mathrm{app}}{ }^{\mathrm{H}}\right)} \\
& M_{0, \mathrm{SS}(\tau)}^{z}=M_{\mathrm{eq}} \sum_{i=1}^{N}\left[p_{i} \frac{1-\exp \left(-\tau / T_{1, i}\right)}{1-f_{0, i} \exp \left(-\tau / T_{1, i}\right)}\right] / \sum_{i=1}^{N} p_{i}
\end{aligned}
$$

Calculation of Uncertainties, Fitting, and Hypothesis Testing. For the magnetization recovery curves (Figure 4a) and the sensitivity curves (Figure 4b), experiments were recorded in replicates. Each series of experiments was recorded by interleaving short and long variable delays in order to make measurements robust against temporally correlated systematic errors, for example due to steady-state effects, RF heating, or drift in instrument conditions.

The uncertainty on the signal intensity was estimated individually for each delay value using the pooled standard deviation. ${ }^{60}$ For a number of replicates $n_{j}\left(n_{j} \geq 3\right)$ and a standard deviation $s_{j}$ among those replicates, the pooled standard deviation $\sigma_{\mathrm{p}}$ is calculated according to eq 16 , where the index $j$ corresponds to each of the three experimental curves $(j=1$, no flip-back; $j=2$, with flip-back; and $j=3$, with flip-down). This uncertainty estimate is used in statistical significance tests and to depict error bars in Figure 4. 


$$
\sigma_{\mathrm{p}}=\sqrt{\left(\sum_{j=1}^{3}\left(n_{j}-1\right) s_{j}{ }^{2}\right) /\left(\sum_{j=1}^{3}\left(n_{j}-1\right)\right)}
$$

The individual curves i, ii, and iii (recovery curve, Figure 4a; or sensitivity curve, Figure $4 \mathrm{~b}$ ) are fitted as a group with either of two models: (A) recovery following a monoexponential behavior (eqs 9 and 14); (B) recovery modeled as the sum of two exponential components (eqs 10 and 15). The following parameters are extracted for each of the exponential components: the fraction of this component relative to the total proton pool $p_{i} / \sum p_{i}$, the longitudinal ${ }^{1} \mathrm{H}$ relaxation time, $T_{1, i}$, and the amount of remaining ${ }^{1} \mathrm{H}$ magnetization which is recovered by the flipback pulse $f_{0, i}$. Values for $M_{\text {eq }}, p_{i}, T_{1, i}$ and $f_{0, i}$ are obtained by minimization of the residual sum of squares between calculated and experimental values $\left(\mathrm{SS}_{\text {lack of fit }}\right)$.

The validity of the fit was assessed by computing the $F$ score, which compares $\mathrm{SS}_{\text {lack of fit }}$ to the sum of squares due to pure experimental error $\left(S_{\text {pure error }}\right)$ estimated from the deviation of replicate measurements. An $F$ score larger than the critical $F$ value indicates that the lack of fit is unlikely to be due to chance alone. Furthermore, since the monoexponential model $\mathrm{A}$ is nested within the multiexponential model B, we use the F-test to determine whether model $\mathrm{B}$ explains the experimental significantly better than model A or whether the reduction in $\mathrm{SS}_{\text {lack of fit }}$ merely results from the increase in the number of parameters.

The uncertainty of the fitted parameters (Table 2) was estimated with a Monte Carlo procedure. The Monte Carlo data point $y_{k}^{\mathrm{MC}}$ is generated from the experimental data point $y_{k}^{\exp }$ using eq 17 , where $\sigma_{k}$ is the uncertainty of this data point as described earlier and $\varepsilon$ is drawn randomly from a normal distribution of mean zero and standard deviation of one. The fitting procedure was repeated for $>20,000$ iterations, and the uncertainty of a parameter is taken as the standard deviation of this parameter in the Monte Carlo fitting results.

$$
y_{k}^{\mathrm{MC}}=y_{k}^{\exp }+\sigma_{k} \varepsilon
$$

\section{RESULTS}

Efficient ${ }^{1} \mathrm{H}$ Spin Locking and ${ }^{1} \mathrm{H}-{ }^{13} \mathrm{C}$ Decoupling Obtained at Ultrafast MAS Using Low-Power RF. In order to obtain a maximal amount of ${ }^{1} \mathrm{H}$ magnetization after a proton-decoupled CP experiment, dephasing has to be avoided during cross-polarization and heteronuclear decoupling.

It has been previously demonstrated that spin-locked ${ }^{1} \mathrm{H}$ magnetization is preserved for longer times at ultrafast MAS under specific ranges of RF nutation frequencies, $\nu_{1}^{1} \mathrm{H} .53,54$ Here, we measured the decay of spin-lock ${ }^{1} \mathrm{H}$ magnetization in uniformly $\left[{ }^{13} \mathrm{C},{ }^{15} \mathrm{~N}\right]$-labeled glutamine at $65 \mathrm{kHz}$ MAS (Figure 1a) using the pulse sequence presented in Figure 2a. As ultrafast MAS strongly reduces homonuclear ${ }^{1} \mathrm{H}$ dipolar couplings, the resonance conditions around $\nu_{1}^{1} \mathrm{H} \approx(1 / 2) \nu_{\mathrm{r}}$ (double-quantum homonuclear rotary resonance, 2Q-HOR$\left.\mathrm{ROR}^{61}\right)$ and $\nu_{1}^{1} \mathrm{H} \approx \nu_{\mathrm{r}}\left(\right.$ rotary resonance $\left.{ }^{62,63}\right)$ are narrowed considerably. However, higher order recoupling conditions ${ }^{53}$ occur at $\nu_{1}^{1} \mathrm{H} \approx(1 / 4) \nu_{\mathrm{r}},(1 / 3) \nu_{\mathrm{r}}$, and $(3 / 2) \nu_{\mathrm{r}}$. Efficient spin lock is obtained at low power when $\nu_{1}^{1} \mathrm{H}$ is in the range of $0.07-0.21$ times the MAS frequency, $\nu_{\mathrm{r}}$. At high power, efficient spin lock is obtained with $\nu_{1}^{1} \mathrm{H} \approx 1.3 \nu_{\mathrm{r}}$ and with the $\nu_{1}^{1} \mathrm{H} / \nu_{\mathrm{r}}$ ratio in the ranges of 1.6-1.8 and 2.3-2.65. Very few differences in decay behavior are observed between different ${ }^{13} \mathrm{C}$ sites. We measured a rotatingframe ${ }^{1} \mathrm{H}$ longitudinal relaxation time, $\mathrm{T}_{1 \rho}=13.9 \pm 0.4 \mathrm{~ms}$, from a
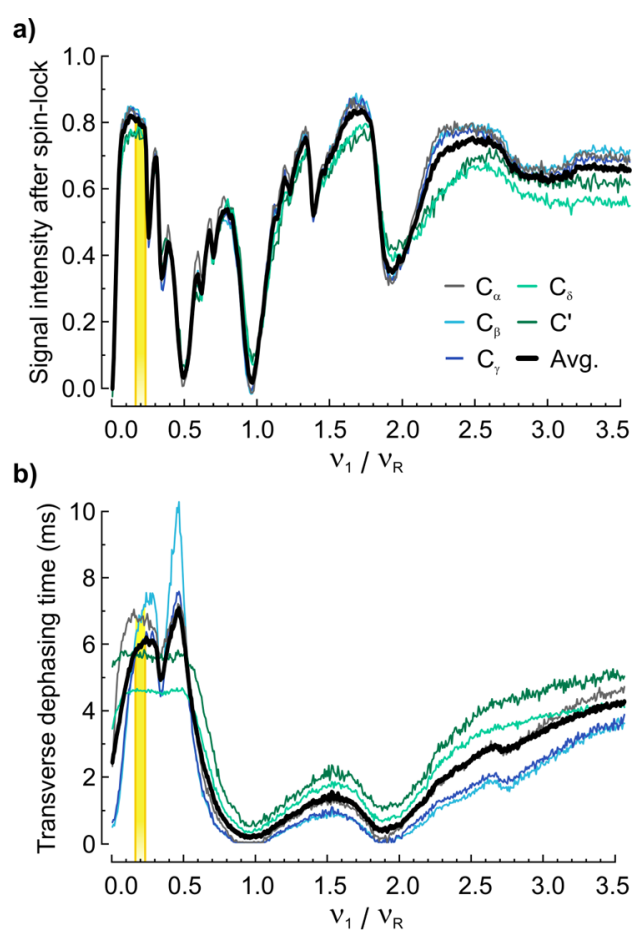

Figure 1. Low-power $\mathrm{cw}$ irradiation allows efficient ${ }^{1} \mathrm{H}$ spin lock and ${ }^{1} \mathrm{H}-{ }^{13} \mathrm{C}$ decoupling at $65 \mathrm{kHz}$ MAS in the range of $\mathrm{RF}$ frequency from $\nu_{1}^{1} \mathrm{H} \approx 0.14 \nu_{\mathrm{r}}$ to $0.21 \nu_{\mathrm{r}}$, as highlighted by yellow rectangles. The average signal intensities or dephasing times are represented as thick lines. (a) ${ }^{1} \mathrm{H}$ spin lock efficiency. The total signal intensity was detected on ${ }^{13} \mathrm{C}$ resonances after $2.5 \mathrm{~ms}$ of ${ }^{1} \mathrm{H}$ spin lock in glutamine as a function of the spin-lock frequency $\nu_{1, \mathrm{SL}}^{1}$. Intensities were normalized against a reference experiment with the spin-lock block omitted. (b) Decoupling efficiency. The Lorentzian broadening of the glutamine ${ }^{13} \mathrm{C}$ resonance line shapes is expressed as a transverse dephasing time $T_{2}{ }^{\prime}(\mathrm{ms})$ for a series of decoupling RF frequency $\nu_{1, \mathrm{dec}} \mathrm{H}$

spin-lock decay curve (Supporting Information Figure S1b) recorded at a nutation frequency of $13.4 \mathrm{kHz}$ at the $\nu_{1}^{\mathrm{H}} \approx 0.21 \nu_{\mathrm{r}}$ condition.

It has also been demonstrated previously that continuous lowpower irradiation produces efficient heteronuclear decoupling at ultrafast MAS. ${ }^{4,64}$ We acquired the $\mathrm{CP}$ spectrum of glutamine at $65 \mathrm{kHz}$ varying the RF frequency $\nu_{1}^{1} \mathrm{H}$ during $\mathrm{cw}$ decoupling (Figure $2 \mathrm{~b}$ ). In addition to the intrinsic relaxation time, $T_{2}$, of transverse ${ }^{13} \mathrm{C}$ coherences, residual terms of the heteronuclear dipolar coupling due to imperfect decoupling contribute to the total homogeneous line-width $\Delta^{\prime}$. This line width is usually measured in the context of a spin-echo, ${ }^{65}$ with an associated transverse dephasing time $T_{2}{ }^{\prime}=\left(\pi \Delta^{\prime}\right)^{-1}$. We quantified the decoupling efficiency (Figure $1 \mathrm{~b}$ ) by determining the Lorentzian broadening of the carbon line shapes $f_{\mathrm{L}}$ and estimating an associated transverse dephasing time $T_{2}{ }^{\prime} \approx\left(\pi f_{L}\right)^{-1}$. In this case, ${ }^{13} \mathrm{C}$ line shapes exhibit different behaviors depending on their number of attached protons. For carbonyls ${ }^{13} \mathrm{C}\left(\mathrm{C}^{\prime}\right.$ and $\left.\mathrm{C}_{\delta}\right)$, efficient decoupling is obtained for $\nu_{1}^{1} \mathrm{H} / \nu_{\mathrm{r}}$ ratios in the range of $0.06-0.50$. A strong recoupling condition occurs at $\nu_{1}^{1} \mathrm{H}=(1 / 3) \nu_{\mathrm{r}}$ for methylene carbons $\left(\mathrm{C}_{\beta}\right.$ and $\left.\mathrm{C}_{\gamma}\right)$, requiring at least three dipolar-coupled protons, ${ }^{66}$ which is practically undetected in nonprotonated carbons and attenuated for $\mathrm{C}_{\alpha}$. For protonated carbons, the best decoupling condition occurs around $\nu_{1}^{1} \mathrm{H} \approx$ $0.46 \nu_{\mathrm{r}}$; however, nearly as efficient decoupling can be obtained in the low-power regime with $\nu_{1}^{1} \mathrm{H}$ ranging from $0.14 \nu_{\mathrm{r}}$ to $0.3 \nu_{\mathrm{r}}$. Both regions of efficient ${ }^{1} \mathrm{H}-{ }^{13} \mathrm{C}$ decoupling correspond to purely 
a) Spin-lock efficiency

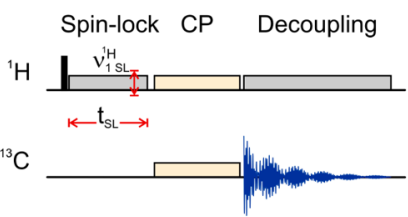

b) Decoupling efficiency
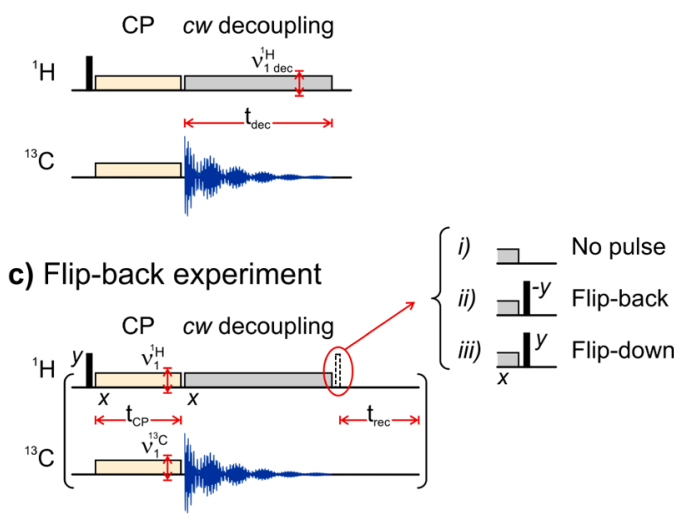

d) Magnetization recovery

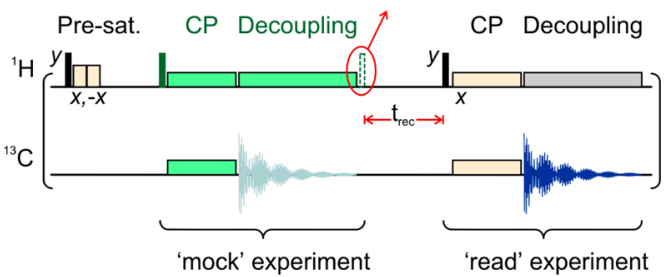

Figure 2. Solid-state NMR pulse schemes for measurement of (a) ${ }^{1} \mathrm{H}$ spin-lock efficiency (Figure 1a), (b) ${ }^{1} \mathrm{H}-{ }^{13} \mathrm{C}$ decoupling efficiency (Figure 1b), (c) sensitivity curve (Figure $4 b$ ) and signal enhancement (Figure 5), and (d) ${ }^{1} \mathrm{H}$ remaining magnetization (Figure 3 ) and recovery curve (Figure 4a). In panel c, the signal intensity is measured on ${ }^{13} \mathrm{C}$ after a SOCP experiment to aliphatic carbons $\left(\mathrm{C}_{\alpha} / \mathrm{C}_{\beta} / \mathrm{C}_{\gamma}\right)$ as a function of the recycling delay. A high number of dummy scans were applied prior to recording to ensure measurement in the steady-state regime. In panel d, to ensure a constant initial amount of magnetization, protons are presaturated followed by a recycling delay. To know the recovery behavior after a CP-decoupling experiment (Figure 4a), a "mock" SOCP experiment is executed, containing either the flip-back pulse, the flipdown pulse, or no pulse. The amount of magnetization regained after a given recovery delay $\left(t_{\mathrm{rec}}\right)$ is then measured by the "read" experiment, a SOCP to aliphatic carbons. To know the remaining ${ }^{1} \mathrm{H}$ magnetization after a CP-decoupling experiment (Figure 3), a "mock" experiment is carried and the remaining ${ }^{1} \mathrm{H}$ magnetization is measured immediately (without flip-back) by a second identical read experiment. Magnetization transferred to ${ }^{13} \mathrm{C}$ by the mock experiment is filtered out by phase cycling. The source code for all employed pulse programs and composite pulse decoupling programs is available in the Supporting Information.

homonuclear ${ }^{1} \mathrm{H}-{ }^{1} \mathrm{H}$ recoupling conditions, the 2Q-HORROR condition ${ }^{61}$ at $\nu_{1}^{1} \mathrm{H} \approx(1 / 2) \nu_{\mathrm{r}}$ and a four-spin condition ${ }^{53}$ at $\nu_{1}^{1} \mathrm{H} \approx$ $(1 / 4) \nu_{\mathrm{r}}$, suggesting that cw decoupling performance is strongly enhanced by self-decoupling, ${ }^{67,68}$ where I-I recoupling conditions promote fast flip-flop fluctuations between I spins which enhances the averaging of I-S dipolar couplings. ${ }^{69,70}$

It is thus possible to find a range of ${ }^{1} \mathrm{H}$ RF frequencies which fulfills both requirements of efficient decoupling and magnetization preservation. This range is situated between $\nu_{1}^{1} \mathrm{H} \approx 0.14 \nu_{\mathrm{r}}$ and $0.21 \nu_{\mathrm{r}}$ (yellow rectangles, Figure 1 ). We thus selected a proton irradiation frequency of $13.4 \mathrm{kHz}\left(\nu_{1}^{1} \mathrm{H} \approx 0.21 \nu_{\mathrm{r}}\right)$ to be used in low-power CP schemes.

${ }^{1} \mathrm{H}$ Magnetization Well-Preserved after Cross-Polarization and Decoupling. A series of low-power and high-power $\mathrm{CP}$ schemes were tested in order to obtain ${ }^{1} \mathrm{H}-{ }^{13} \mathrm{C}$ transfer: bandselective double-quantum (DQ) $\mathrm{CP}{ }^{71}$ second-order crosspolarization (SOCP), ${ }^{53}$ amplitude-modulated SOCP (MOD$\mathrm{CP}),{ }^{54}$ and high-power $\mathrm{CP},{ }^{72,73}$ using either double-quantum or zero-quantum (ZQ) Hartmann-Hahn conditions. ${ }^{3}$ The very efficient ${ }^{1} \mathrm{H}$ spin-lock conditions previously mentioned $\left(\nu_{1}{ }^{1} \mathrm{H} \approx\right.$ $\left.0.21 \nu_{\mathrm{r}}\right)$ was employed for SOCP and MOD-CP. The contact time, RF field strengths, and carrier offsets were optimized for each CP scheme and are listed in Table 1 . Schemes based on the

Table 1. Conditions for Cross-Polarization at $65 \mathrm{kHz}$ MAS and Remaining ${ }^{1} \mathrm{H}$ Magnetization

\begin{tabular}{|c|c|c|c|c|}
\hline cross-polarization condition & $\nu_{1}^{1} \mathrm{H}, \mathrm{kHz}$ & $\nu_{1}^{13} \mathrm{C}, \mathrm{kHz}$ & $\begin{array}{l}\text { contact } \\
\text { time, } \mathrm{ms}\end{array}$ & $\begin{array}{l}\text { residual } \\
{ }^{1} \mathrm{H}, \%\end{array}$ \\
\hline \multicolumn{5}{|l|}{ band-selective conditions } \\
\hline $\begin{array}{l}\text { SOCP to aliphatic } \\
\left(\mathrm{C}_{\alpha}, \mathrm{C}_{\beta}, \mathrm{C}_{\gamma}\right)\end{array}$ & 13.4 & 13.1 & 1.5 & 41.9 \\
\hline $\begin{array}{l}\text { SOCP to carbonyl } \\
\left(\mathrm{C}_{\delta}, \mathrm{C}^{\prime}\right)\end{array}$ & 13.4 & 13.2 & 6.0 & 37.3 \\
\hline $\begin{array}{l}\text { DQ CP }(n=1) \text { to } \\
\text { aliphatic }\end{array}$ & 52.5 & $11.2^{a}$ & 1.4 & 28.6 \\
\hline $\begin{array}{l}\mathrm{DQCP}(n=1) \text { to } \\
\text { carbonyl }\end{array}$ & 52.5 & $11.6^{a}$ & 1.0 & 28.4 \\
\hline \multicolumn{5}{|l|}{ broadband conditions } \\
\hline MOD-CP & 13.4 & $28.0^{b}$ & 4.5 & 28.6 \\
\hline DQCP $(n=2)$ & 87.5 & $35.1^{a}$ & 1.3 & 36.3 \\
\hline $\mathrm{ZQCP}(n=1)$ & 116.1 & $48.4^{a}$ & 2.8 & 23.4 \\
\hline
\end{tabular}

${ }^{a} \mathrm{~A} 100-80 \%$ ramp of the carbon RF amplitude ${ }^{74}$ was applied for nonSOCP-based schemes. The average $(90 \%){ }^{13} \mathrm{C} \mathrm{RF}$ frequency is reported. ${ }^{b}$ For MOD-CP, a cosine modulation of frequency $13.5 \mathrm{kHz}$ was applied to the carbon RF amplitude. To preserve the $n=0$ matching condition for both excitation bands, the ${ }^{13} \mathrm{C}$ RF frequency must be doubled. ${ }^{54}$

second-order cross-polarization mechanism provide the highest amount of signal (Figure 3). In the case of broadband schemes, a compromise was chosen to avoid disparate intensities for nuclei that have distinct transfer or relaxation properties. This kind of compromise is generally pursued in the case where all types of functional groups must be characterized with equal importance.

We then estimated, using the pulse sequence presented in Figure $2 \mathrm{~d}$, the amount of remaining ${ }^{1} \mathrm{H}$ magnetization after one of the following pulse elements (Figure 3): (i) a ${ }^{1} \mathrm{H}$ spin lock, (ii) cross-polarization, and (iii) cross-polarization followed by $\mathrm{cw}$ decoupling. For all tested CP schemes, we find that a significant amount of the initial polarization, from $42 \%$ to $24 \%$, remains available on protons after cross-polarization and cw decoupling, (Figure 3, red spectra). The amount of polarization remaining after each step can be easily predicted from spin-lock decay (Supporting Information Figure S1b) and transfer to carbon as discussed in Magnetization Recovery: A General Method for Sensitivity Enhancement Compatible with Several Cross-Polarization Schemes. These results indicate that proton-decoupled $\mathrm{CP}$ experiments at very fast MAS are suitable candidates for sensitivity enhancement by ${ }^{1} \mathrm{H}$ magnetization recovery.

We then focused subsequent investigations on the SOCP to aliphatic ${ }^{13} \mathrm{C}$ transfer scheme, as it offers the largest percentage of remaining magnetization and highest transfer efficiency. 


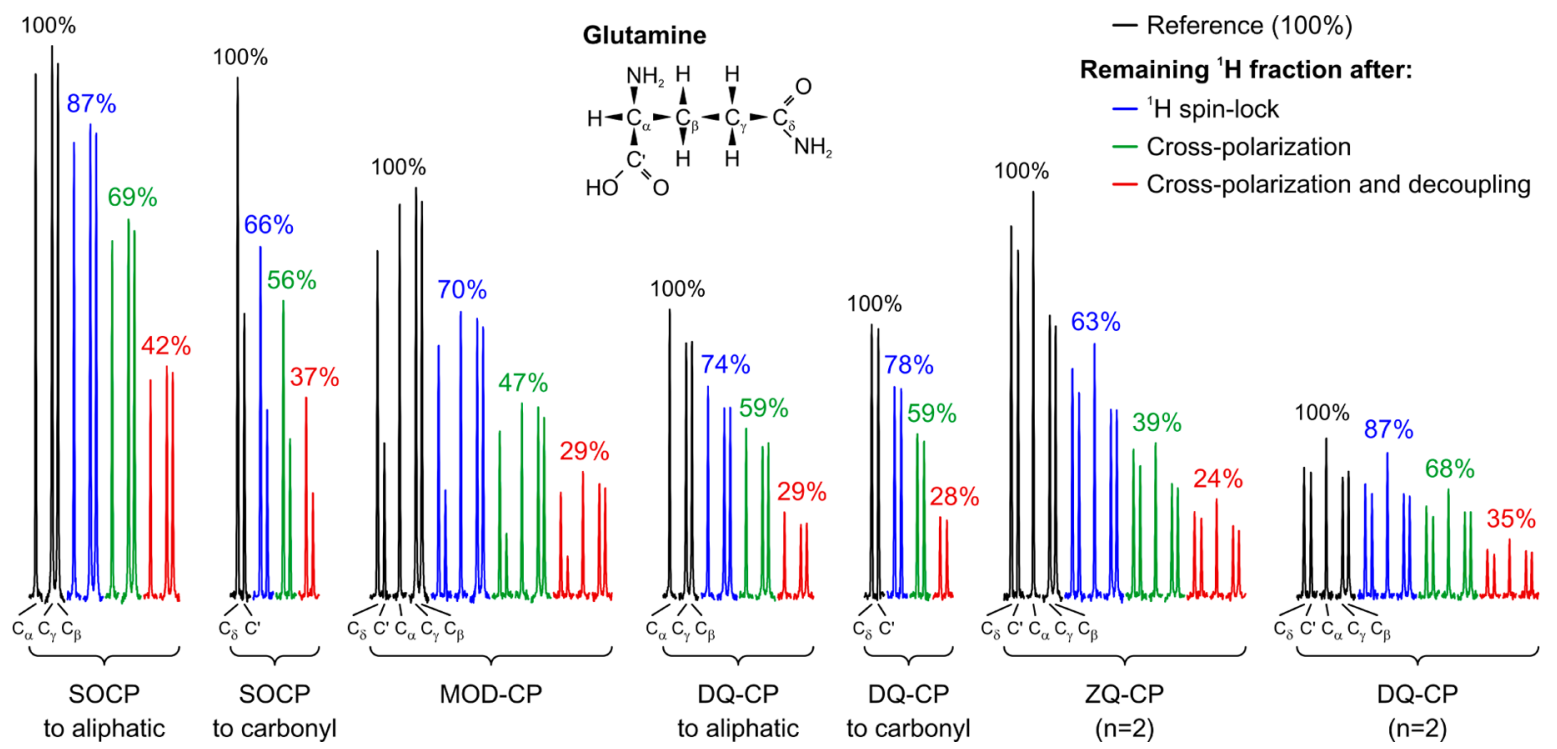

Figure 3. Significant amounts of ${ }^{1} \mathrm{H}$ magnetization remain after different steps of the CP-decoupling experiment. For series of low-power and highpower $\mathrm{CP}$ conditions, different elements of the $\mathrm{CP}$-decoupling experiment were executed (blue, only ${ }^{1} \mathrm{H}$ spin lock; green, only $\mathrm{CP}$; red, $\mathrm{CP}$ followed by ${ }^{1} \mathrm{H}$ decoupling) in a "mock" experiment as described in Figure $2 \mathrm{~d}$. The remaining levels of ${ }^{1} \mathrm{H}$ magnetization were then immediately measured (without flip-back) by employing a second "read" experiment with identical CP conditions. The decoupling time employed in the mock experiment had a duration of $8 \mathrm{~ms}$. The RF field frequencies and contact times for each CP scheme are detailed in Table 1.
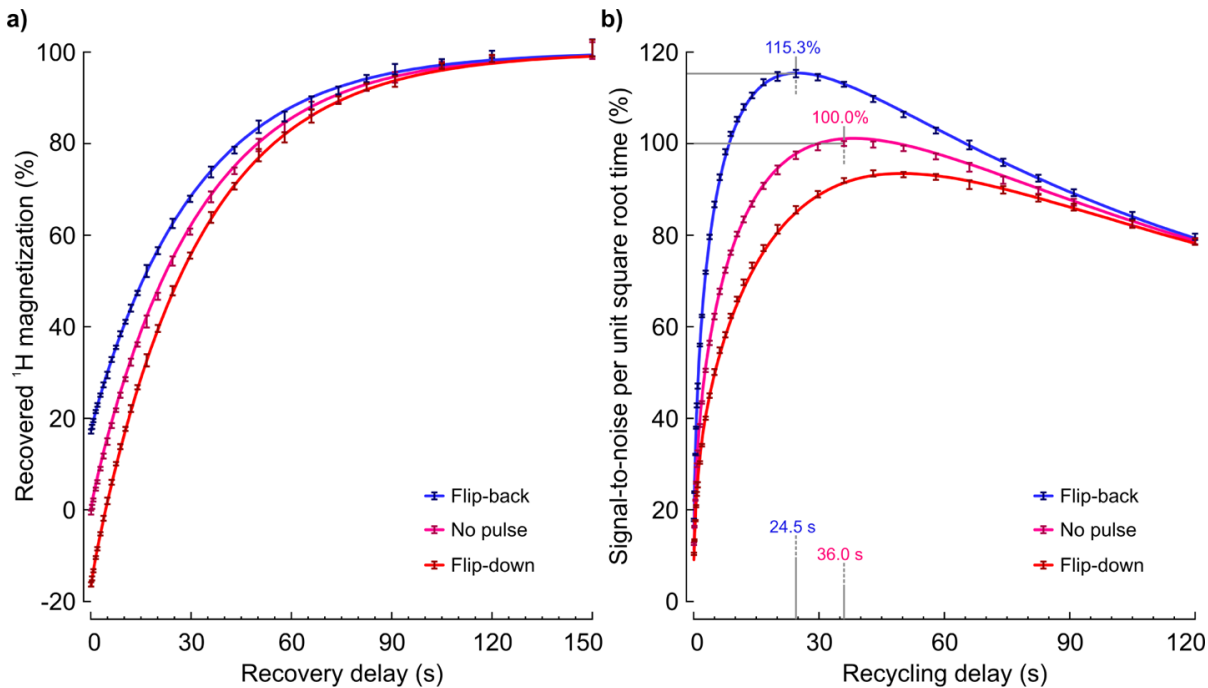

Figure 4. Recovery curve and sensitivity curve in glutamine at $65 \mathrm{kHz}$ MAS following a SOCP to aliphatic carbons with low-power cw decoupling. (a) Time course of ${ }^{1} \mathrm{H}$ magnetization recovery recorded with the pulse scheme in Figure $2 \mathrm{~d}$. The amount of recovered ${ }^{1} \mathrm{H}$ magnetization is larger if the experiment finishes with a flip-back pulse (blue), compared to no final pulse (magenta), or a flip-down pulse (red). Magnetization amounts are normalized to the total equilibrium magnetization as extracted from the fit. (b) Sensitivity (signal-to-noise per unit square root time) measured using the pulse scheme in Figure 2c. The sensitivity is normalized to the maximal experimental sensitivity in the absence of flip-back pulse. Maximal sensitivity is obtained by using the flip-back pulse (blue, $115.3 \pm 0.8 \%, 24.5 \mathrm{~s}$ recycling delay), compared to the reference experiment with no recovery of ${ }^{1} \mathrm{H}$ magnetization (magenta, $100.0 \pm 0.5 \%, 36.0 \mathrm{~s}$ recycling delay) or experiments with flip-down (red). For panels a and $\mathrm{b}$, solid lines correspond to the solution of the least-squares fit when all experimental data are fitted together with the recovery modeled as the sum of two exponential components, model B (eqs 10 and 15).

Enhanced Magnetization Recovery Leading to Higher Achievable Sensitivity and Shorter Optimal Recycling Time. We monitored the recovery of magnetization following a proton-decoupled SOCP to aliphatic ${ }^{13} \mathrm{C}$ experiment (Figure 4a). Three sets of experiments were recorded: (i) reference experiments, where no pulse were applied following decoupling; (ii) flip-back experiments, with a $90^{\circ}$ pulse placing ${ }^{1} \mathrm{H}$ magnetization back to $+\hat{I}_{z}$; or (iii) flip-down experiments, with a $90^{\circ}$ pulse placing ${ }^{1} \mathrm{H}$ down along $-\hat{I}_{z}$. A significantly larger amount of magnetization was recovered in the presence of the flip-back pulse compared to the reference, for all delays up to $58 \mathrm{~s}$ (Student's $t$ test, 95\% confidence level).

In order to assess the sensitivity gains provided by the flip-back pulse, the signal-to-noise ratio per unit square root time $(\mathrm{S} / \mathrm{N})_{t}$ was measured as a function of the interscan delay for the three sets of experiments (Figure $4 b$ ). The measurement (Figure 2c) is performed in the steady state so as to be independent of the number of scans already performed. 
Compared to the reference experiments, flip-back experiments yield a higher $(\mathrm{S} / \mathrm{N})_{t}$ for all measured interscan delays up to $120 \mathrm{~s}$; however, when the remaining ${ }^{1} \mathrm{H}$ magnetization is placed along $-\hat{I}_{z}$ in the flip-down experiments, the sensitivity is reduced for all interscan delays up to $91 \mathrm{~s}$ (Student's $t$-test, 95\% confidence level). Accordingly, the highest obtainable $(\mathrm{S} / \mathrm{N})_{t}$ was found to be improved from $100.0 \pm 0.5 \%$ in the reference experiment to $115.3 \pm 0.8 \%$ with the flip-back pulse.

Another advantage provided by the fastened recovery is the reduction of the recycling delay. If sensitivity is not a limiting factor, a $(\mathrm{S} / \mathrm{N})_{t}$ of $100 \%$ can be reached in only $8.1 \mathrm{~s}$ using the flip-back pulse, compared to $36.0 \mathrm{~s}$ in the reference experiment. More than four times as many transients are recorded in the same amount of time, which is beneficial for experiments with extended phase cycling. The shorter duration can also allow smaller increments in the indirect dimension of multidimensional experiments. The interscan duration is also shortened in the case of sensitivity-limited experiments: the interscan duration yielding the highest sensitivity is $36.0 \mathrm{~s}$ for the reference experiment compared to $24.5 \mathrm{~s}$ when the flip-back pulse is applied, representing a reduction of more than $30 \%$ in interscan delay.

\section{DISCUSSION}

Magnetization Recovery: A General Method for Sensitivity Enhancement Compatible with Several Cross-Polarization Schemes. From the results presented in Figure 3, it can be seen that all tested CP schemes are able to preserve significant amounts of ${ }^{1} \mathrm{H}$ magnetization. This is attributed in part to the selection of efficient ${ }^{1} \mathrm{H}$ spin-lock conditions, either at low power $\left(\nu_{1}^{1} \mathrm{H} \approx 0.21 \nu_{\mathrm{r}}\right)$ for SOCPbased schemes or at high power for other schemes $\left(\nu_{1_{1}}^{1} \mathrm{H} \approx\right.$ $0.81 \nu_{\mathrm{r}}$ for DQCP $n=1, \nu_{1} \mathrm{H} \approx 1.35 \nu_{\mathrm{r}}$ for DQCP $n=2$, and $\nu_{1} \mathrm{H} \approx$ $1.79 \nu_{\mathrm{r}}$ for ZQCP $\left.n=1\right)$. The bulk ${ }^{1} \mathrm{H}$ magnetization remaining after ${ }^{1} \mathrm{H}$ spin-lock pulse (blue spectra in Figure 3 ) follows eq 18, where $t_{\mathrm{SL}}$ is the spin-lock duration and $T_{1 \rho}$ is the ${ }^{1} \mathrm{H}$ longitudinal relaxation time in the rotating frame.

$$
M_{\left(t_{\mathrm{SL}}\right)}=M_{0} \exp \left(-t_{\mathrm{SL}} / T_{1 \rho}\right)
$$

Using the experimentally determined value of $T_{1 \rho} \approx 13.9 \mathrm{~ms}$ determined at the $\nu_{1}^{1} \mathrm{H} \approx 0.21 \nu_{\mathrm{r}}$ condition (Supporting Information Figure $\mathrm{S} 1 \mathrm{~b}$ ) and a spin-lock duration of $t_{\mathrm{SL}}=$ $1.5 \mathrm{~ms}$, we calculate a remaining amount of $90 \%$ in agreement with the experimental measure of $87 \%$. The values of ${ }^{1} \mathrm{H} T_{1 \rho}$ for other ${ }^{1} \mathrm{H}$ spin-lock frequencies can be estimated from the spinlock efficiency curve (Figure 1a). The calculated amounts after spin lock have a deviation of less than $5 \%$ compared to the measured values (Figure 3, blue spectra) except for ZQ CP which has approximately $20 \%$ less magnetization than calculated.

In addition to spin-lock decay, the ${ }^{1} \mathrm{H}$ magnetization is reduced during $\mathrm{CP}$ due to transfer of polarization from ${ }^{1} \mathrm{H}$ to ${ }^{13} \mathrm{C}$, which is illustrated by the difference between ${ }^{1} \mathrm{H}$ spin lock (Figure 3, blue spectra) and CP experiments (Figure 3, green spectra). We observe that, for the different $\mathrm{CP}$ schemes, the proportion of ${ }^{1} \mathrm{H}$ magnetization contributing to the transfer is roughly proportional to the number of carbons excited by the $\mathrm{CP}$ scheme, this proportion increasing by $7 \pm 2 \%$ for each additional carbon.

Based on the characteristics of the spin lock, $T_{1 \rho} \approx 13.9 \mathrm{~ms}$ during $8 \mathrm{~ms}$, we calculate that $56 \%$ of the magnetization will be preserved through the final $\mathrm{cw}$ decoupling. Indeed, considering all seven $\mathrm{CP}$ schemes, we measured that the intensities after $\mathrm{CP}$ decoupling (Figure 3, red spectra) have a ratio of $57 \pm 7 \%$ compared to CP alone (Figure 3, green spectra).

As initial polarization is preserved for all CP schemes, with amounts ranging from $42 \%$ to $24 \%$ after CP-decoupling experiments, the effect of the flip-back pulse was assessed for three recycling delays: $0.5,2.0$, and $20 \mathrm{~s}$ (Figure 5). Compared to the reference without flip-back, the flip-back provided an increase in the total signal ranging from $24 \%$ to $50 \%$ with a 0.5 s recycling delay, $24 \%$ to $48 \%$ with a 2.0 s recycling delay, and $14 \%$ to $21 \%$ for a $20.0 \mathrm{~s}$ recycling delay.

The intensities of individual peaks are conserved between the reference and the flip-back experiment and do not vary significantly when changing the recycling delay. The recovery enhancement method is thus potentially applicable for quantitative experiments and multidimensional experiments. A high correlation is also observed between the enhancement factor (Figure 5) and the amount of remaining ${ }^{1} \mathrm{H}$ magnetization as determined in Figure 3.

Preserving Large Magnetization Amounts Is Principal Contribution to Sensitivity Enhancement but Magnetization Sharing Also a Marginal Contribution at Fast Repetition Rates. Several mechanisms can be proposed to explain the increased sensitivity. The simplest mechanism is that using the flip-back pulse brings the system closer to its thermal equilibrium state, without any change in ${ }^{1} \mathrm{H} T_{1}$ relaxation rates. An alternative hypothesis states that the flip-back pulse accelerates the apparent longitudinal relaxation rate of protons directly contributing to heteronuclear transfer. ${ }^{48}$

In this second mechanism, contributing and noncontributing protons act as separate magnetization pools, each with their respective population size, their longitudinal relaxation time, $T_{1}$, and their residual polarization level prior to recovery. According to the concept of spin temperature, ${ }^{75}$ noncontributing protons have a higher population of spins in the low-energy state after $\mathrm{cw}$ decoupling and are thus "colder", while contributing protons are close to saturation, thus "warmer", and have nearly a positive infinite temperature. A heat flow is established between the different proton pools when a perturbation can mediate an exchange of spin order between the magnetization pools. In the case considered, protonproton dipolar couplings between the two reservoirs mediate this exchange, leading to the establishment of a quasi-equilibrium steady state after a certain time period. ${ }^{76}$ Although discussed here in thermodynamics terms, this exchange is a coherent process which can be monitored via hole burning experiments. ${ }^{77}$

We investigated the presence and the extent of this magnetization sharing mechanism in our system using two diagnostics: the detection of multiexponential recovery behavior and the comparison of extracted recovery rates to the bulk longitudinal relaxation rate measured by inversion-recovery (Table 2 and Supporting Information Figure S1a). The multiexponential behavior of the recovery and sensitivity curves arises as contributing protons benefit from both magnetization sharing and longitudinal relaxation at short recovery delays but only benefit from longitudinal relaxation of both pools in the limit of very long recovery delay once a steady state has been established. $^{78}$

The experimental data were fitted considering two models of magnetization recovery: in model $\mathrm{A}$, a monoexponential recovery behavior was assumed; in model $\mathrm{B}$, the recovery followed a sum of two exponentials. In addition to recording the sensitivity and recovery curves with no pulse or a flip-back following cw decoupling, we also recorded a series of experiments where the remaining magnetization is flipped "down" 
a) Recycling delay: $0.5 \mathrm{~s}$
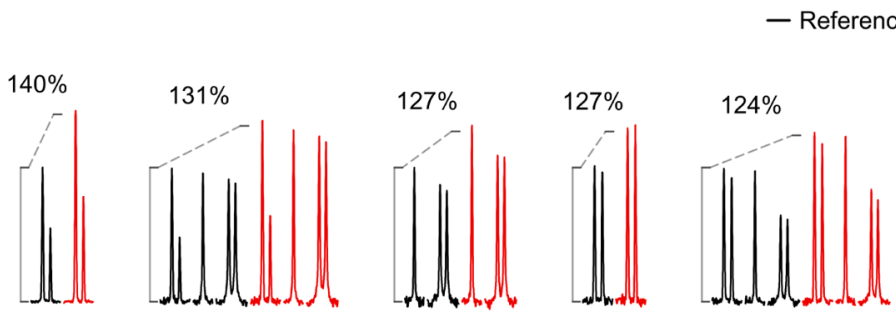

- Flip-back
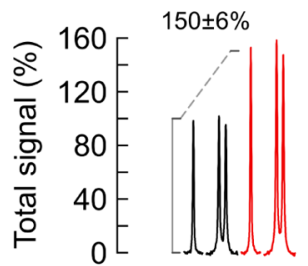

b) Recycling delay: $2.0 \mathrm{~s}$
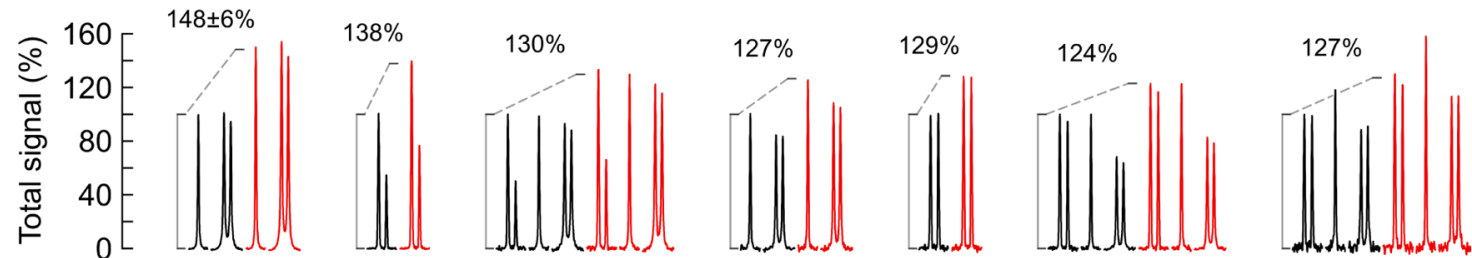

c) Recycling delay: $20 \mathrm{~s}$
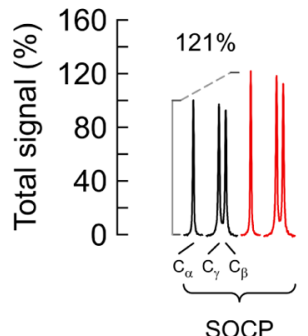

to aliphatic

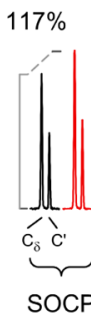

SOCP

to carbonyl
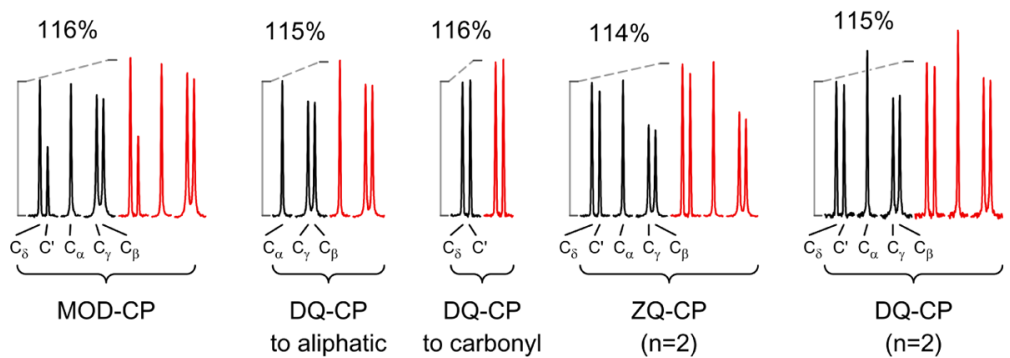

Figure 5. Enhancement in the fast pulsing limit. The total signal intensities obtained for CP-decoupling experiments with flip-back pulse (red spectra) are compared to reference experiments recorded without flip-back pulse (normalized to $100 \%$ total signal intensities, black spectra). The conditions employed are the same as for the series of CP schemes presented in Figure 3 and Table 1. Significant enhancements are obtained with the recycling delay in the fast pulsing regime ( $\mathrm{a}$ and $\mathrm{b}$ ) as well as in the regime providing maximal sensitivity $(\mathrm{c})$.

Table 2. Extraction of Relaxation Parameters from Recovery and Sensitivity Curves

\begin{tabular}{|c|c|c|c|c|c|}
\hline model & $\begin{array}{c}\text { component } \\
\text { population, } \%\end{array}$ & relaxation time, $T_{1}^{1} \mathrm{H}, \mathrm{s}$ & residual ${ }^{1} \mathrm{H}$ magnetization, $\%$ & $F_{\text {score }}^{a}$ & $F_{\text {critical }}{ }^{b}$ \\
\hline \multicolumn{6}{|l|}{ fit of recovery curve (Figure 4a) } \\
\hline one exponential component & $(100)$ & $30.9 \pm 0.26$ & $34.0 \pm 0.25$ & 0.64 & 1.50 \\
\hline \multirow[t]{2}{*}{ two exponential components } & $81 \pm 28$ & $37 \pm 27$ & $24 \pm 13$ & 0.32 & 1.51 \\
\hline & $19 \pm 28$ & $16 \pm 8.7$ & $11 \pm 13$ & & \\
\hline \multicolumn{6}{|l|}{ fit of sensitivity curve (Figure $4 b$ ) } \\
\hline one exponential component & $(100)$ & $28.4 \pm 0.10$ & $34.1 \pm 0.16$ & 10.33 & 1.48 \\
\hline two exponential components & $\begin{array}{l}98.8 \pm 0.15 \\
1.2 \pm 0.15\end{array}$ & $\begin{array}{l}30.9 \pm 0.24 \\
1.3 \pm 0.19\end{array}$ & $\begin{array}{l}33.9 \pm 0.19 \\
25 \pm 4.2\end{array}$ & 0.87 & 1.49 \\
\hline \multicolumn{6}{|l|}{ simultaneous fit } \\
\hline one exponential component & $(100)$ & $29.06 \pm 0.09$ & $34.4 \pm 0.14$ & 2.41 & 1.33 \\
\hline \multirow[t]{2}{*}{ two exponential components } & $98.7 \pm 0.13$ & $31.3 \pm 0.21$ & $34.1 \pm 0.16$ & 0.58 & 1.33 \\
\hline & $1.3 \pm 0.13$ & $1.4 \pm 0.16$ & $0.9 \pm 0.52$ & & \\
\hline \multicolumn{6}{|l|}{ fit of inversion recovery ( Figure $S 1 a^{c}$ ) } \\
\hline one exponential component & & $32 \pm 2.2$ & & & \\
\hline
\end{tabular}

${ }^{a} \mathrm{An} \mathrm{F}_{\text {score }}>\mathrm{F}_{\text {critical }}$ value indicates that the lack of fit is unlikely to be due to chance alone. ${ }^{b}$ Critical $\mathrm{F}$ values were computed at the $99 \%$ confidence level. ${ }^{c}$ Supporting Information.

along the $-\hat{I}_{z}$ axis. By forcing a higher population of spins to occupy the high-energy (unfavorable) state, both proton pools acquire a negative spin temperature. The noncontributing protons have a larger population difference and are considered "warmer" than the contributing protons which are close to saturation. The inclusion of the flip-down series makes it easier to detect a multiexponential behavior of the magnetization recovery, since the flow of heat is inverted relative to the flip-back 
series of experiment and magnetization sharing between proton pools slows down the recovery of contributing protons at short recycling delays instead of fastening it.

For the recovery curves (Figure 4a), both model A (monoexponential recovery) and model B (sum of two exponentials), are compatible with the data $\left(F_{\text {score }}<F_{\text {critiral }}\right.$, $99 \%$ confidence level); however, the fit is significantly better with model B (F-test, $99 \%$ confidence level). For the sensitivity curves (Figure $4 \mathrm{~b}$ ), the experimental data are only compatible when the recovery is modeled as the sum of two exponential components (model B) but is incompatible with a monoexponential recovery behavior (model A).

We carried out a Monte Carlo procedure to estimate the uncertainty of extracted parameters. This procedure reveals that the fitting of recovery data alone is insufficient to allow the precise determination of parameters. The fitting of data to a sum of exponentials is indeed a well-known badly conditioned problem, such that small variations in the data can result in large variations of the extracted parameters. ${ }^{79,80}$ On the contrary, the fitting of the sensitivity data alone was sufficient to extract welldefined parameters with low standard deviation obtained in the Monte Carlo routine.

In order to obtain a global model of the data, we carry a simultaneous fit of recovery and sensitivity data using the twoexponential components (model B). The resulting fit is compatible with both sets of data and yields well-defined parameters (Table 2, simultaneous fit). A simultaneous fit of recovery and sensitivity data with three exponential components could provide an improved agreement with the data; however, the two main exponential components present the same features as found in model B, and the extracted parameters are not welldefined.

The fit with model B uncovers the presence of a major $(98.7 \pm$ $0.13 \%)$ and a minor $(1.3 \pm 0.13 \%)$ population component. While the minor component follows a quick apparent relaxation $\left(T_{1}=1.4 \pm 0.16 \mathrm{~s}\right)$, the major component relaxes at a slower rate $\left(T_{1}=31.3 \pm 0.21 \mathrm{~s}\right)$. This relaxation time is statistically compatible with the longitudinal relaxation time of bulk protons $\left(T_{1}=32 \pm 2.2 \mathrm{~s}\right)$ as measured by a standard inversion-recovery experiment (Supporting Information Figure S1). The amount of preserved proton magnetization for the major component is quite large $(34.1 \pm 0.16 \%)$ and is sufficient alone to explain the sensitivity enhancement and shortening of the optimal recycling time compared to the reference experiment. Indeed, neglecting the minor component only marginally affects the maximal obtainable sensitivity with flip-back, which decreases to $114.3 \%$ from $115.4 \%$, or the expected optimal recycling time, which increases from 24.8 to $25.9 \mathrm{~s}$. However, the sensitivity obtained at very short recycling delays is more severely affected, with a reduction of over $14 \%$ for recycling delay of $1.0 \mathrm{~s}$ and below.

Recommended Settings and Implementation Strategy for Unlabeled Compounds. Sufficiently long acquisition times must be employed to avoid truncation of the signal of the FID, which would lead to broadening of the NMR resonances. This reduction in the spectral resolution is reflected at short acquisition times by a decrease of the ${ }^{13} \mathrm{C}$ peak heights in the reference experiment (Figure 6, red curve). On the other hand, the flip-back pulse provides an improvement in sensitivity for all studied decoupling times up to $30 \mathrm{~ms}$ of decoupling (Figure 6, blue curve). However, the magnitude of the enhancement diminishes at longer decoupling times as the amount of preserved ${ }^{1} \mathrm{H}$ magnetization is reduced. In this study, we selected a decoupling time of $8 \mathrm{~ms}$ (highlighted in Figure 6) as it provides

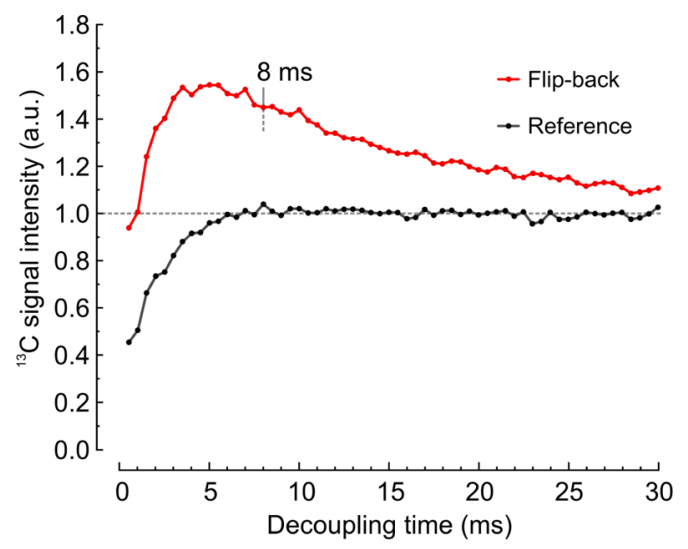

Figure 6. Sensitivity gains and resolution as a function of decoupling time. A series of SOCP to aliphatic carbons CP-decoupling experiments were recorded with flip-back (red curve) and with no pulse applied after decoupling (black curve), with a 2 s recycle delay. For all experiments, the ${ }^{13} \mathrm{C}$ receiver was open for $30 \mathrm{~ms}$ and the duration of the ${ }^{1} \mathrm{H} \mathrm{cw}$ decoupling pulse was varied from 0 to $30 \mathrm{~ms}$. The signal intensities at the central peak frequency were summed for the three aliphatic carbon peaks $\left(\mathrm{C}_{\alpha} / \mathrm{C}_{\beta} / \mathrm{C}_{\gamma}\right)$. At short decoupling times $(<8 \mathrm{~ms})$, an incomplete FID is acquired, causing broadening of the Fourier-transformed peaks and reduction in peak height of the reference spectrum (without flip-back, black curve). The reference experiment reaches its maximal intensity with decoupling time $\geq 8 \mathrm{~ms}$, indicating the absence of resolution loss due to FID truncation. The decoupling time of $8 \mathrm{~ms}$ employed in the CP-decoupling experiments of Figures $3-5$ is indicated.

a high enhancement and does not lead to any significant peak broadening of the reference spectrum. Further gains in sensitivity can be obtained at shorter decoupling times in the special case where resolution can be compromised, for example in the indirect dimensions of multidimensional experiments or if peak positions are known and well-separated.

For the optimization of parameters for the measurement of natural abundance spectra, we recommend the following procedure:

(i) Prior to the experiment, calibrate the $\mathrm{X}$ channel power level on a uniformly labeled sample with similar physical characteristics to the sample of interest. Estimate the required acquisition time and optimize the cross-polarization conditions. For example, adjust the attenuation level $(\mathrm{dB})$ to have a ${ }^{13} \mathrm{C} \mathrm{RF}$ strength of $\nu_{1}^{13} \mathrm{C} \approx 0.20 \nu_{\mathrm{r}}$ for SOCP.

(ii) Using the natural abundance sample, determine the bulk ${ }^{1} \mathrm{H} T_{1}$ relaxation rate. The ${ }^{1} \mathrm{H} T_{1}$ can be determined using proton-detected inversion-recovery $\left(180^{\circ}\right.$-delay- $\left.90^{\circ}\right)$ or saturation-recovery experiment (saturation-delay-90 $\left.{ }^{\circ}\right){ }^{81}$ To ensure a constant initial magnetization, a recycling delay of $5 T_{1, \text { app }}^{1} \mathrm{H}$ must be employed or a presaturation element must be applied prior to recycling.

(iii) Calibrate the ${ }^{1} \mathrm{H}$ channel power level and adjust the attenuation level $(\mathrm{dB})$ to have a ${ }^{1} \mathrm{H}$ RF strength of $\nu_{1}^{1} \approx 0.20 \nu_{\mathrm{r}}$ (e.g., $13 \mathrm{kHz} \mathrm{RF}$ at $65 \mathrm{kHz}$ MAS).

(iv) Determine the ${ }^{1} \mathrm{H}$ spin-lock decay $T_{1 \rho}$ at that power level and determine the remaining amount of ${ }^{1} \mathrm{H}$ magnetization $f_{0}$. While the optimal recycling delay without flip-back is $\tau_{\mathrm{opt}}^{\text {no }} \approx$ $1.25 T_{1, \mathrm{app}}^{\mathrm{H}}$, the optimal recycling delay with flip-back pulse is given by $\tau_{\mathrm{opt}}^{\mathrm{FB}} \approx\left(1-f_{0}\right)\left(1.25 T_{1, \mathrm{app}}^{\mathrm{H}}\right)$ using the experimental values of $T_{1, \mathrm{app}}^{1} \mathrm{H}$ and the remaining amount $f_{0}$.

\section{CONCLUSION}

The present study demonstrates that the preservation of ${ }^{1} \mathrm{H}$ magnetization during all employed pulse elements leads to significant sensitivity gains and shortening of the recycling delay. 
The sensitivity gains do not rely on selective excitation and magnetization sharing, rather on careful conservation of ${ }^{1} \mathrm{H}$ magnetization, preserving more than $55 \%$ of the initial polarization amount after $8 \mathrm{~ms}$ of decoupling. This makes our method additionally compatible with broadband experiments and uniformly labeled materials, in contrast to application conducted at low MAS $(<20 \mathrm{kHz})$ and fast MAS $(20-40 \mathrm{kHz})$.

Sensitivity enhancement could be obtained with a large variety of CP schemes. The intensity of individual peaks was retained in the enhanced spectra, enabling the use of our method to be employed for quantitative applications. Remaining amounts for each CP scheme could be predicted on the basis of the selected spin-lock conditions and number of excited carbons. The enhancement ratio provided by the flip-back is highly related to the amount of remaining ${ }^{1} \mathrm{H}$ magnetization. With the SOCP to aliphatic CP scheme, the optimal sensitivity was increased to $115.3 \%$ of the initial value $(100 \%)$ and the optimal recycling delay was shortened to $68 \%$ of its original duration. The sensitivity enhancement is combined with the gains already provided by the SOCP scheme, which increases the signal to 1.5 times the amount delivered by high-power $\mathrm{CP}$ schemes. ${ }^{53}$ Broadband ${ }^{13} \mathrm{C}$ excitation can be realized by the MOD-CP scheme which also provide a higher signal compared to high-power CP schemes. ${ }^{54}$ Using these two combinations, completely low-RF power sensitivity-enhanced experiments can be realized.

In contrast to the fast MAS regime $(20-40 \mathrm{kHz})$ where highpower decoupling is required, low-power $\mathrm{cw}$ irradiation can provide efficient heteronuclear decoupling at ultrafast MAS due to the reversal of the averaging process ${ }^{64}$ and has been used for the recording of the resolved spectrum of uniformly labeled human superoxide dismutase. ${ }^{4}$ The performance of low-power heteronuclear decoupling has been enhanced by rapid phase modulation of the irradiation, for instance with the decoupling sequences low-power $\mathrm{XiX},{ }^{82}$ low-power $\mathrm{TPPM}^{83}$ swept lowpower TPPM, ${ }^{8}$ low-amplitude PISSARO, ${ }^{84}$ and amplitudemodulated low-power XiX. ${ }^{85}$ Although these sequences offer longer ${ }^{13} \mathrm{C}$ coherence lifetimes approaching the high-RF power extreme limit reached with microcoils, ${ }^{86}$ the time scale of their phase modulation and pulse lengths is on the same order as the rotor period. The rapid pulse phase transitions lead to decoherence of the ${ }^{1} \mathrm{H}$ magnetization, making these decoupling sequences incompatible with magnetization recovery sensitivity enhancement. It can be envisioned however that ultrafast MAS decoupling schemes which can afford longer pulse durations such as the phase-alternated XiX scheme ${ }^{82}$ or the rCW schemes ${ }^{87,88}$ can be adapted in order to preserve high amounts of ${ }^{1} \mathrm{H}$ magnetization after decoupling.

The sensitivity enhancement strategy can readily be extended to more sophisticated pulse schemes and multidimensional experiments. Indeed, several pulse sequence elements employed at ultrafast $\mathrm{MAS}^{3}$ contain lengthy blocks of $\mathrm{cw}$ irradiation on ${ }^{1} \mathrm{H}$, for example PAR, ${ }^{89}$ PAIN, ${ }^{90}$ and MIRROR, ${ }^{91,92}$ which can additionally serve as a low-power ${ }^{1} \mathrm{H}$ spin lock. In this case, the conditions of low decay described here can be employed. On the other hand, several recoupling elements such as DREAM, ${ }^{93}$ radio frequency driven recoupling (RFDR), ${ }^{94}$ and ${ }^{13} \mathrm{C}-{ }^{15} \mathrm{~N} \mathrm{CP}^{19,20}$ do not require any ${ }^{1} \mathrm{H}$ irradiation at ultrafast MAS such that ${ }^{1} \mathrm{H}$ magnetization can be stored along $+\hat{I}_{z}$ during those periods. As an example, the RELOAD-CP experiment ${ }^{33}$ could be easily modified to benefit from the two sensitivity enhancement strategies, ${ }^{1} \mathrm{H}$ recovery and ${ }^{13} \mathrm{C}$ magnetization sharing. Similarly, the complete set of low-power experiments for protein assignment ${ }^{20}$ and the pulse sequences used for the PACC approach ${ }^{19}$ can be adapted for ${ }^{1} \mathrm{H}$ magnetization recovery.

Although a multiexponential behavior is clearly detected from the fitting of recovery and sensitivity data, ${ }^{1} \mathrm{H}$ magnetization sharing did not play a large role in sensitivity enhancement for the system studied here. As the proton spin-lock condition employed for SOCP-based experiments is close to a purely homonuclear recoupling condition involving four coupled ${ }^{1} \mathrm{H}$ spins, ${ }^{53}$ it is likely that rapid proton-proton mixing already occurs in the rotating frame during cross-polarization and decoupling. The magnetization transferred to carbon in SOCP-based experiments is thus already enhanced by the contribution of remote protons.

The processes of longitudinal proton relaxation and proton spin diffusion are severely truncated at ultrafast spinning and under high external magnetic fields. The fast ${ }^{1} \mathrm{H}-{ }^{1} \mathrm{H}$ mixing occurring during decoupling explains why the magnetization sharing mechanism did not contribute to recovery enhancement except at very short recycling delays, since a quasi-equilibrium state is already established during the course of decoupling. This observation is supported by the highly similar recovery behaviors observed for the seven different CP schemes. This knowledge could be of high importance in the following years. It was reported that at even faster MAS rates $(110 \mathrm{kHz})$, large variations in ${ }^{1} \mathrm{H} T_{1}$ exist between different atoms within a molecule, such that the apparent recovery rate is accelerated by applying RFDR during the recovery. ${ }^{95}$ The two approaches are compatible and could be combined to overcome further sensitivity boundaries in multidimensional experiments, by preserving bulk proton magnetization through efficient low-power irradiation and accelerating recovery through RF-induced proton-proton mixing.

\section{ASSOCIATED CONTENT}

\section{Supporting Information}

Measurements of bulk ${ }^{1} \mathrm{H}$ longitudinal relaxation and ${ }^{1} \mathrm{H}$ spinlock decay (Figure S1) and text and charts containing the source code for the pulse sequence programs employed in this work presenting full phase cycles, description of the parameters, and instructions for using the programs on TopSpin 2.x and 3.x systems and including pulse sequences for (a) ${ }^{1} \mathrm{H}$ spin-lock efficiency and ${ }^{1} \mathrm{H}-{ }^{13} \mathrm{C}$ decoupling efficiency experiments, (b) flip-back experiment and associated code for composite pulse decoupling programs, and (c) measuring the remaining magnetization and magnetization recovery curves and full references from this work with more than 10 authors. This material is available free of charge via the Internet at http://pubs.acs.org.

\section{AUTHOR INFORMATION}

\section{Corresponding Author}

*E-mail: demers@fmp-berlin.de. Tel.: +49 (0)30 94793287. Fax: +49 (0)3094793 169 .

\section{Present Addresses}

$\dagger$ (J.-P.D., A.L.) Department of Molecular Biophysics, LeibnizInstitut für Molekulare Pharmakologie (FMP), 13125 Berlin, Germany.

$\ddagger$ (V.V.) Department of Chemistry, IISER Thiruvananthapuram, 695016, Kerala, India.

\section{Author Contributions}

The manuscript was written through contributions of all authors. All authors have given approval to the final version of the manuscript.

\section{Notes}

The authors declare no competing financial interest. 


\section{ACKNOWLEDGMENTS}

We thank the Max Planck Society, the Leibniz-Institut für Molekulare Pharmakologie, the Deutsche Forschungsgemeinschaft (Emmy Noether Fellowship to A.L.), and the European Union Seventh Framework Program under Grant Agreement 261863 (Bio-NMR) as well as the Natural Sciences and Engineering Research Council of Canada (postgraduate scholarship to J.-P.D.) for financial support. We thank Dr. Veniamin Chevelkov, Dr. Davood Bakhtiari, Dr. Antoine Loquet, and Dr. Donghan Lee for helpful discussions as well as Brigitta Angerstein for technical assistance.

\section{ABBREVIATIONS}

$\mathrm{CP}$, cross-polarization; DQ, double-quantum; 2Q-HORROR, double-quantum homonuclear rotary resonance; FID, free induction decay; NMR, nuclear magnetic resonance; MAS, magic-angle spinning; MOD-CP, amplitude-modulated SOCP; $\mathrm{RF}$, radio frequency; RFDR, radio frequency driven recoupling; SOCP, second-order cross-polarization; $\mathrm{ZQ}$, zero-quantum

\section{REFERENCES}

(1) Lesage, A. Recent Advances in Solid-State NMR Spectroscopy of Spin $\mathrm{I}=1 / 2$ Nuclei. Phys. Chem. Chem. Phys. 2009, 11, 6876-6891.

(2) Samoson, A.; Tuherm, T.; Past, J.; Reinhold, A.; Heinmaa, I.; Anupõld, T.; Smith, M. E.; Pike, K. J. Fast Magic-Angle Spinning: Implications; John Wiley \& Sons: Hoboken, NJ, USA, 2010.

(3) Demers, J.-P.; Chevelkov, V.; Lange, A. Progress in Correlation Spectroscopy at Ultra-fast Magic-Angle Spinning: Basic Building Blocks and Complex Experiments for the Study of Protein Structure and Dynamics. Solid. State. Nucl. Magn. Reson. 2011, 40, 101-113.

(4) Laage, S.; Sachleben, J. R.; Steuernagel, S.; Pierattelli, R.; Pintacuda, G.; Emsley, L. Fast Acquisition of Multi-Dimensional Spectra in SolidState NMR Enabled by Ultra-Fast MAS. J. Magn. Reson. 2009, 196, 133141.

(5) Nadaud, P. S.; Helmus, J. J.; Sengupta, I.; Jaroniec, C. P. Rapid Acquisition of Multidimensional Solid-State NMR Spectra of Proteins Facilitated by Covalently Bound Paramagnetic Tags. J. Am. Chem. Soc. 2010, 132, 9561-9563.

(6) Bertini, I.; Emsley, L.; Lelli, M.; Luchinat, C.; Mao, J. F.; Pintacuda, G.; Ultrafast, M. A. S. Solid-State NMR Permits Extensive ${ }^{13} \mathrm{C}$ and ${ }^{1} \mathrm{H}$ Detection in Paramagnetic Metalloproteins. J. Am. Chem. Soc. 2010, 132, $5558-5559$.

(7) Knight, M. J.; Webber, A. L.; Pell, A. J.; Guerry, P.; Barbet-Massin, E.; Bertini, I.; Felli, I. C.; Gonnelli, L.; Pierattelli, R.; Emsley, L.; et al. Fast Resonance Assignment and Fold Determination of Human Superoxide Dismutase by High-Resolution Proton-Detected SolidState MAS NMR Spectroscopy. Angew. Chem., Int. Ed. 2011, 50, 1169711701.

(8) Lewandowski, J. R.; Sein, J.; Sass, H. J.; Grzesiek, S.; Blackledge, M.; Emsley, L. Measurement of Site-Specific ${ }^{13} \mathrm{C}$ Spin-Lattice Relaxation in a Crystalline Protein. J. Am. Chem. Soc. 2010, 132, 8252-8254.

(9) Tollinger, M.; Sivertsen, A. C.; Meier, B. H.; Ernst, M.; Schanda, P. Site-Resolved Measurement of Microsecond-to-Millisecond Conformational-Exchange Processes in Proteins by Solid-State NMR Spectroscopy. J. Am. Chem. Soc. 2012, 134, 14800-14807.

(10) Paluch, P.; Pawlak, T.; Amoureux, J.-P.; Potrzebowski, M. J. Simple and Accurate Determination of X-H Distances under Ultra-Fast MAS NMR. J. Magn. Reson. 2013, 233, 56-63.

(11) Bertini, I.; Emsley, L.; Felli, I. C.; Laage, S.; Lesage, A.; Lewandowski, J. R.; Marchetti, A.; Pierattelli, R.; Pintacuda, G. HighResolution and Sensitivity Through-Bond Correlations in Ultra-Fast Magic Angle Spinning (MAS) Solid-State NMR. Chem. Sci. 2011, 2, 345-348.

(12) Webber, A. L.; Pell, A. J.; Barbet-Massin, E.; Knight, M. J.; Bertini, I.; Felli, I. C.; Pierattelli, R.; Emsley, L.; Lesage, A.; Pintacuda, G. Combination of DQ and ZQ Coherences for Sensitive Through-Bond
NMR Correlation Experiments in Biosolids under Ultra-Fast MAS. ChemPhysChem 2012, 13, 2405-2411.

(13) Barbet-Massin, E.; Pell, A. J.; Knight, M. J.; Webber, A. L.; Felli, I. C.; Pierattelli, R.; Emsley, L.; Lesage, A.; Pintacuda, G. ${ }^{13}$ C-Detected Through-Bond Correlation Experiments for Protein Resonance Assignment by Ultra-Fast MAS Solid-State NMR. ChemPhysChem 2013, 14, 3131-3137.

(14) Hou, G.; Yan, S.; Sun, S.; Han, Y.; Byeon, I. L.; Ahn, J.; Concel, J.; Samoson, A.; Gronenborn, A. M.; Polenova, T. Spin Diffusion Driven by R-Symmetry Sequences: Applications to Homonuclear Correlation Spectroscopy in MAS NMR of Biological and Organic Solids. J. Am. Chem. Soc. 2011, 133, 3943-3953.

(15) Lafon, O.; Trebosc, J.; Hu, B.; De Paepe, G.; Amoureux, J.-P. Observing ${ }^{13} \mathrm{C}-{ }^{13} \mathrm{C}$ Connectivities at High Magnetic Fields and Very High Spinning Frequencies. Chem. Commun. (Cambridge, U. K.) 2011, 47, 6930-6932.

(16) Hou, G.; Byeon, I. J. L.; Jinwoo, A.; Gronenborn, A. M.; Polenova, T. Recoupling of Chemical Shift Anisotropy by R-Symmetry Sequences in Magic Angle Spinning NMR Spectroscopy. J. Chem. Phys. 2012, 137, No. 134201.

(17) Shen, M.; Hu, B. W.; Lafon, O.; Trebosc, J.; Chen, Q.; Amoureux, J. P. Broadband Finite-Pulse Radio-Frequency-Driven Recoupling (fpRFDR) with (XY8) $4^{1}$ Super-Cycling for Homo-Nuclear Correlations in Very High Magnetic Fields at Fast and Ultra-Fast MAS Frequencies. J. Magn. Reson. 2012, 223, 107-119.

(18) Wickramasinghe, N. P.; Shaibat, M. A.; Jones, C. R.; Casabianca, L. B.; de Dios, A. C.; Harwood, J. S.; Ishii, Y. Progress in ${ }^{13} \mathrm{C}$ and ${ }^{1} \mathrm{H}$ Solid-State Nuclear Magnetic Resonance for Paramagnetic Systems under Very Fast Magic Angle Spinning. J. Chem. Phys. 2008, 128, No. 052210 .

(19) Wickramasinghe, N. P.; Parthasarathy, S.; Jones, C. R.; Bhardwaj, C.; Long, F.; Kotecha, M.; Mehboob, S.; Fung, L. W. M.; Past, J.; Samoson, A.; et al. Nanomole-Scale Protein Solid-State NMR by Breaking Intrinsic ${ }^{1} \mathrm{H} \mathrm{T}_{1}$ Boundaries. Nat. Methods 2009, 6, 215-218.

(20) Vijayan, V.; Demers, J. P.; Biernat, J.; Mandelkow, E.; Becker, S.; Lange, A. Low-Power Solid-State NMR Experiments for Resonance Assignment under Fast Magic-Angle Spinning. ChemPhysChem 2009, 10, 2205-2208.

(21) Sun, S.; Yan, S.; Guo, C.; Li, M.; Hoch, J. C.; Williams, J. C.; Polenova, T.; Time-Saving, A. Strategy for MAS NMR Spectroscopy by Combining Nonuniform Sampling and Paramagnetic Relaxation Assisted Condensed Data Collection. J. Phys. Chem. B 2012, 116, 13585-13596.

(22) Parthasarathy, S.; Nishiyama, Y.; Ishii, Y. Sensitivity and Resolution Enhanced Solid-State NMR for Paramagnetic Systems and Biomolecules under Very Fast Magic Angle Spinning. Acc. Chem. Res. 2013, 46, 2127-2135.

(23) Zhou, D. H.; Shah, G.; Cormos, M.; Mullen, C.; Sandoz, D.; Rienstra, C. M. Proton-Detected Solid-State NMR Spectroscopy of Fully Protonated Proteins at $40 \mathrm{kHz}$ Magic-Angle Spinning. J. Am. Chem. Soc. 2007, 129, 11791-11801.

(24) Marchetti, A.; Jehle, S.; Felletti, M.; Knight, M. J.; Wang, Y.; Xu, Z.-Q.; Park, A. Y.; Otting, G.; Lesage, A.; Emsley, L.; et al. Backbone Assignment of Fully Protonated Solid Proteins by ${ }^{1} \mathrm{H}$ Detection and Ultrafast Magic-Angle-Spinning NMR Spectroscopy. Angew. Chem., Int. Ed. 2012, 51, 10756-10759.

(25) Barbet-Massin, E.; Pell, A. J.; Jaudzems, K.; Franks, W. T.; Retel, J. S.; Kotelovica, S.; Akopjana, I.; Tars, K.; Emsley, L.; Oschkinat, H.; et al. Out-and-Back ${ }^{13} \mathrm{C}-{ }^{13} \mathrm{C}$ Scalar Transfers in Protein Resonance Assignment by Proton-Detected Solid-State NMR under Ultra-Fast MAS. J. Biomol. NMR 2013, 56, 379-386.

(26) Linser, R.; Bardiaux, B.; Higman, V.; Fink, U.; Reif, B. Structure Calculation from Unambiguous Long-Range Amide and Methyl ${ }^{1} \mathrm{H}-{ }^{1} \mathrm{H}$ Distance Restraints for a Microcrystalline Protein with MAS Solid-State NMR Spectroscopy. J. Am. Chem. Soc. 2011, 133, 5905-5912.

(27) Lewandowski, J. R.; Dumez, J. N.; Akbey, U.; Lange, S.; Emsley, L.; Oschkinat, H. Enhanced Resolution and Coherence Lifetimes in the Solid-State NMR Spectroscopy of Perdeuterated Proteins under 
Ultrafast Magic-Angle Spinning. J. Phys. Chem. Lett. 2011, 2, 22052211.

(28) Zhou, D. H. H.; Nieuwkoop, A. J.; Berthold, D. A.; Comellas, G.; Sperling, L. J.; Tang, M.; Shah, G. J.; Brea, E. J.; Lemkau, L. R.; Rienstra, C. M. Solid-State NMR Analysis of Membrane Proteins and Protein Aggregates by Proton Detected Spectroscopy. J. Biomol. NMR 2012, 54, 291-305.

(29) Asami, S.; Szekely, K.; Schanda, P.; Meier, B. H.; Reif, B. Optimal Degree of Protonation for ${ }^{1} \mathrm{H}$ Detection of Aliphatic Sites in Randomly Deuterated Proteins as a Function of the MAS Frequency. J. Biomol. NMR 2012, 54, 155-168.

(30) Chevelkov, V.; Habenstein, B.; Loquet, A.; Giller, K.; Becker, S.; Lange, A. Proton-Detected MAS NMR Experiments Based on Dipolar Transfers for Backbone Assignment of Highly Deuterated Proteins. J. Magn. Reson. 2014, 242, 180-188.

(31) Xiang, S.; Chevelkov, V.; Becker, S.; Lange, A. Towards Automatic Protein Backbone Assignment using Proton-Detected 4D Solid-State NMR Data. J. Biomol. NMR 2014, 60, 85-90.

(32) Barbet-Massin, E.; Pell, A. J.; Retel, J. S.; Andreas, L. B.; Jaudzems, K.; Franks, W. T.; Nieuwkoop, A. J.; Hiller, M.; Higman, V.; Guerry, P.; et al. Rapid Proton-Detected NMR Assignment for Proteins with Fast Magic Angle Spinning. J. Am. Chem. Soc. 2014, 136, 12489-12497.

(33) Barnes, A. B.; De Paepe, G.; van der Wel, P. C. A.; Hu, K. N.; Joo, C. G.; Bajaj, V. S.; Mak-Jurkauskas, M. L.; Sirigiri, J. R.; Herzfeld, J.; Temkin, R. J.; et al. High-Field Dynamic Nuclear Polarization for Solid and Solution Biological NMR. Appl. Magn. Reson. 2008, 34, 237-263.

(34) Maly, T.; Debelouchina, G. T.; Bajaj, V. S.; Hu, K. N.; Joo, C. G.; Mak-Jurkauskas, M. L.; Sirigiri, J. R.; van der Wel, P. C. A.; Herzfeld, J.; Temkin, R. J. Dynamic Nuclear Polarization at High Magnetic Fields. J. Chem. Phys. 2008, 128, No. 052211.

(35) Lopez, J. J.; Kaiser, C.; Asami, S.; Glaubitz, C. Higher Sensitivity through Selective ${ }^{13} \mathrm{C}$ Excitation in Solid-State NMR Spectroscopy. J. Am. Chem. Soc. 2009, 131, 15970-15971.

(36) Banigan, J. R.; Traaseth, N. J. Utilizing Afterglow Magnetization from Cross-Polarization Magic-Angle-Spinning Solid-State NMR Spectroscopy To Obtain Simultaneous Heteronuclear Multidimensional Spectra. J. Phys. Chem. B 2012, 116, 7138-7144.

(37) Banigan, J. R.; Gayen, A.; Traaseth, N. J. Combination of ${ }^{15} \mathrm{~N}$ Reverse Labeling and Afterglow Spectroscopy for Assigning Membrane Protein Spectra by Magic-Angle-Spinning Solid-State NMR: Application to the Multidrug Resistance Protein EmrE. J. Biomol. NMR 2013, 55, 391-399.

(38) Martineau, C.; Decker, F.; Engelke, F.; Taulelle, F. Parallelizing Acquisitions of Solid-State NMR Spectra with Multi-Channel Probe and Multi-Receivers: Applications to Nanoporous Solids. Solid. State Nucl. Magn. Reson. 2013, 55-56, 48-53.

(39) Gopinath, T.; Veglia, G. 3D DUMAS: Simultaneous Acquisition of Three-Dimensional Magic Angle Spinning Solid-State NMR Experiments of Proteins. J. Magn. Reson. 2012, 220, 79-84.

(40) Gopinath, T.; Veglia, G. Dual Acquisition Magic-Angle Spinning Solid-State NMR-Spectroscopy: Simultaneous Acquisition of Multidimensional Spectra of Biomacromolecules. Angew. Chem., Int. Ed. 2012, 51, 2731-2735.

(41) Gopinath, T.; Veglia, G. Orphan Spin Operators Enable the Acquisition of Multiple 2D and 3D Magic Angle Spinning Solid-State NMR Spectra. J. Chem. Phys. 2013, 138, No. 184201.

(42) Lamley, J. M.; Lewandowski, J. R. Simultaneous Acquisition of Homonuclear and Heteronuclear Long-Distance Contacts with TimeShared Third Spin Assisted Recoupling. J. Magn. Reson. 2012, 218, 3034.

(43) Diercks, T.; Daniels, M.; Kaptein, R. Extended Flip-Back Schemes for Sensitivity Enhancement in Multidimensional HSQC-Type Outand-Back Experiments. J. Biomol. NMR 2005, 33, 243-259.

(44) Pervushin, K.; Vogeli, B.; Eletsky, A. Longitudinal ${ }^{1} \mathrm{H}$ Relaxation Optimization in TROSY NMR Spectroscopy. J. Am. Chem. Soc. 2002, 124, 12898-12902.

(45) Schanda, P.; Brutscher, B. Very Fast Two-Dimensional NMR Spectroscopy for Real-Time Investigation of Dynamic Events in
Proteins on the Time Scale of Seconds. J. Am. Chem. Soc. 2005, 127, 8014-8015.

(46) Deschamps, M.; Campbell, I. D. Cooling Overall Spin Temperature: Protein NMR Experiments Optimized for Longitudinal Relaxation Effects. J. Magn. Reson. 2006, 178, 206-211.

(47) Tegenfeldt, J.; Haeberlen, U. Cross Polarization in Solids with Flip-Back of I-Spin Magnetization. J. Magn. Reson. (1969-1992) 1979, $36,453-457$.

(48) Giffard, M.; Bardet, M.; Bersch, B.; Covès, J.; Hediger, S. Impact of Selective Excitation on Carbon Longitudinal Relaxation: Towards Fast Solid-State NMR Techniques. J. Magn. Reson. 2009, 200, 153-160.

(49) Lupulescu, A.; Frydman, L. Sensitizing Solid State Nuclear Magnetic Resonance of Dilute Nuclei by Spin-Diffusion Assisted Polarization Transfers. J. Chem. Phys. 2011, 135, No. 134202.

(50) Saito, K.; Martineau, C.; Fink, G.; Taulelle, F. Flip-back, an Old Trick to Face Highly Contrasted Relaxation Times: Application in the Characterization of Pharmaceutical Mixtures by CPMAS NMR. Solid State Nucl. Magn. Reson. 2011, 40, 66-71.

(51) Chevelkov, V.; Giller, K.; Becker, S.; Lange, A. Efficient CO-CA Transfer in Highly Deuterated Proteins by Band-Selective Homonuclear Cross-Polarization. J. Magn. Reson. 2013, 230, 205-211.

(52) Chevelkov, V.; Reif, B. Sensitivity Enhancement in Proton Detected Solid State NMR of Highly Deuterated Proteins by Avoiding Water Saturation. 2014.

(53) Lange, A.; Scholz, I.; Manolikas, T.; Ernst, M.; Meier, B. H. LowPower Cross Polarization in Fast Magic-Angle Spinning NMR Experiments. Chem. Phys. Lett. 2009, 468, 100-105.

(54) Demers, J.-P.; Vijayan, V.; Becker, S.; Lange, A. Tailored LowPower Cross-Polarization under Fast Magic-Angle Spinning. J. Magn. Reson. 2010, 205, 216-223.

(55) Lundgren, J. SPLINEFIT, http://www.mathworks.com/ matlabcentral/fileexchange/13812-splinefit, 2010.

(56) Weideman, J. A. C. Computation of the Complex Error Function. SIAM J. Numer. Anal. 1994, 31, 1497-1518.

(57) Olivero, J. J.; Longbothum, R. L. Empirical Fits to the Voigt Line Width: A Brief Review. J. Quant. Spectrosc. Radiat. Transfer 1977, 17, 233-236.

(58) Ernst, R. R.; Bodenhausen, G.; Wokaun, A. Principles of Nuclear Magnetic Resonance in One and Two Dimensions; Clarendon Press: Oxford, U.K., 1994.

(59) Hiller, S.; Wider, G.; Etezady-Esfarjani, T.; Horst, R.; Wuthrich, K. Managing the Solvent Water Polarization to Obtain Improved NMR Spectra of Large Molecular Structures. J. Biomol. NMR 2005, 32, 61-70.

(60) Olofsson, G.; Angus, S.; Armstrong, G. T.; Kornilov, A. N. Assignment and Presentation of Uncertainties of the Numerical Results of Thermodynamic Measurements. Pure Appl. Chem. 1981, 53, 18051826.

(61) Nielsen, N. C.; Bildsoe, H.; Jakobsen, H. J.; Levitt, M. H. DoubleQuantum Homonuclear Rotary Resonance: Efficient Dipolar Recovery in Magic-Angle Spinning Nuclear Magnetic Resonance. J. Chem. Phys. 1994, 101, 1805-1812.

(62) Levitt, M. H.; Oas, T. G.; Griffin, R. G. Rotary Resonance Recoupling in Heteronuclear Spin Pair Systems. Isr. J. Chem. 1988, 28, $271-282$.

(63) Oas, T. G.; Griffin, R. G.; Levitt, M. H. Rotary Resonance Recoupling of Dipolar Interactions in Solid-State Nuclear MagneticResonance Spectroscopy. J. Chem. Phys. 1988, 89, 692-695.

(64) Ernst, M.; Samoson, A.; Meier, B. H. Low-Power Decoupling in Fast Magic-Angle Spinning NMR. Chem. Phys. Lett. 2001, 348, 293302.

(65) Lesage, A.; Bardet, M.; Emsley, L. Through-Bond Carbon-Carbon Connectivities in Disordered Solids by NMR. J. Am. Chem. Soc. 1999, 121, 10987-10993.

(66) Ernst, M.; Samoson, A.; Meier, B. H. Decoupling and Recoupling using Continuous-Wave Irradiation in Magic-Angle-Spinning SolidState NMR: A Unified Description using Bimodal Floquet Theory. J. Chem. Phys. 2005, 123, No. 10.

(67) Sinning, G.; Mehring, M.; Pines, A. Dynamics of Spin Decoupling in ${ }^{13} \mathrm{C}-{ }^{1} \mathrm{H}$ NMR. Chem. Phys. Lett. 1976, 43, 382-386. 
(68) Mehring, M.; Sinning, G. Dynamics of Heteronuclear Spin Coupling and Decoupling in Solids. Phys. Rev. B 1977, 15, 2519-2532.

(69) Tekely, P.; Palmas, P.; Canet, D. Effect of Proton Spin-Exchange on the Residual ${ }^{13} \mathrm{C}$ MAS NMR Linewidths-Phase-Modulated Irradiation for Efficient Heteronuclear Decoupling in Rapidly Rotating Solids. J. Magn. Reson., Ser. A 1994, 107, 129-133.

(70) De Paepe, G.; Elena, B.; Emsley, L. Characterization of Heteronuclear Decoupling through Proton Spin Dynamics in SolidState Nuclear Magnetic Resonance Spectroscopy. J. Chem. Phys. 2004, 121, 3165-3180.

(71) Laage, S.; Marchetti, A.; Sein, J.; Pierattelli, R.; Sass, H. J.; Grzesiek, S.; Lesage, A.; Pintacuda, G.; Emsley, L. Band-Selective ${ }^{1} \mathrm{H}-{ }^{13} \mathrm{C}$ Cross-Polarization in Fast Magic Angle Spinning Solid-State NMR Spectroscopy. J. Am. Chem. Soc. 2008, 130, 17216-17217.

(72) Pines, A.; Gibby, M. G.; Waugh, J. S. Proton-Enhanced NMR of Dilute Spins in Solids. J. Chem. Phys. 1973, 59, 569-590.

(73) Stejskal, E. O.; Schaefer, J.; Waugh, J. S. Magic-Angle Spinning and Polarization Transfer in Proton-Enhanced NMR. J. Magn. Reson. 1977, 28, 105-112.

(74) Metz, G.; Wu, X. L.; Smith, S. O. Ramped-Amplitude Cross Polarization in Magic-Angle-Spinning NMR. J. Magn. Reson., Ser. A 1994, 110, 219-227.

(75) Goldman, M. Spin Temperature and Nuclear Magnetic Resonance in Solids; Clarendon Press: Oxford, U.K., 1970.

(76) Levitt, M. H.; Suter, D.; Ernst, R. R. Spin Dynamics and Thermodynamics in Solid-State NMR Cross-Polarization. J. Chem. Phys. 1986, 84, 4243-4255.

(77) Roos, M.; Micke, P.; Hempel, G. Monitoring Nuclear Spin-Flip Processes and Measuring Spin-Diffusion Constants via Hole Burning into the Magnetization. Chem. Phys. Lett. 2012, 536, 147-154.

(78) Helms, G. Interaction of Exchange and Differential Relaxation in the Saturation Recovery Behavior of the Binary Spin-Bath Model for Magnetization Transfer. Concept Magn. Reson. A 2006, 28A, 291-298.

(79) De Groen, P.; De Moor, B. The Fit of a Sum of Exponentials to Noisy Data. J. Comput. Appl. Math. 1987, 20, 175-187.

(80) Narayanan, A.; Hartman, J. S.; Bain, A. D. Characterizing Nonexponential Spin-Lattice Relaxation in Solid-State NMR by Fitting to the Stretched Exponential. J. Magn. Reson., Ser. A 1995, 112, 58-65.

(81) Becker, E. D.; Ferretti, J. A.; Gupta, R. K.; Weiss, G. H. Choice of Optimal Parameters for Measurement of Spin-Lattice Relaxation-Times. 2. Comparison of Saturation Recovery, Inversion Recovery, and Fast Inversion Recovery Experiments. J. Magn. Reson. 1980, 37, 381-394.

(82) Ernst, M.; Samoson, A.; Meier, B. H. Low-Power XiX Decoupling in MAS NMR Experiments. J. Magn. Reson. 2003, 163, 332-339.

(83) Kotecha, M.; Wickramasinghe, N. P.; Ishii, Y. Efficient LowPower Heteronuclear Decoupling in ${ }^{13} \mathrm{C}$ High-Resolution Solid-State NMR under Fast Magic Angle Spinning. Magn. Reson. Chem. 2007, 45, S221-S230.

(84) Weingarth, M.; Bodenhausen, G.; Tekely, P. Low-Power Decoupling at High Spinning Frequencies in High Static Fields. J. Magn. Reson. 2009, 199, 238-241.

(85) Agarwal, V.; Tuherm, T.; Reinhold, A.; Past, J.; Samoson, A.; Ernst, M.; Meier, B. H. Amplitude-Modulated Low-Power Decoupling Sequences for Fast Magic-Angle Spinning NMR. Chem. Phys. Lett. 2013, $583,1-7$.

(86) Vasa, S. K.; Janssen, H.; Van Eck, E. R. H.; Kentgens, A. P. M. High-Resolution Solid-State ${ }^{13} \mathrm{C} \mu$ MAS NMR with Long Coherence Life Times. Phys. Chem. Chem. Phys. 2011, 13, 104-106.

(87) Vinther, J. M.; Nielsen, A. B.; Bjerring, M.; van Eck, E. R. H.; Kentgens, A. P. M.; Khaneja, N.; Nielsen, N. C. Refocused ContinuousWave Decoupling: A New Approach to Heteronuclear Dipolar Decoupling in Solid-State NMR Spectroscopy. J. Chem. Phys. 2012, 137, No. 214202.

(88) Equbal, A.; Paul, S.; Mithu, V. S.; Madhu, P. K.; Nielsen, N. C. Efficient Heteronuclear Decoupling in MAS Solid-State NMR using Non-Rotor-Synchronized rCW Irradiation. J. Magn. Reson. 2014, 246, 104-109.
(89) Lewandowski, J. R.; De Paepe, G.; Eddy, M. T.; Struppe, J.; Maas, W.; Griffin, R. G. Proton Assisted Recoupling at High Spinning Frequencies. J. Phys. Chem. B 2009, 113, 9062-9069.

(90) Lewandowski, J. R.; De Paepe, G.; Griffin, R. G. Proton Assisted Insensitive Nuclei Cross Polarization. J. Am. Chem. Soc. 2007, 129, 728729 .

(91) Scholz, I.; Huber, M.; Manolikas, T.; Meier, B. H.; Ernst, M. MIRROR Recoupling and its Application to Spin Diffusion under Fast Magic-Angle Spinning. Chem. Phys. Lett. 2008, 460, 278-283.

(92) Scholz, I.; Meier, B. H.; Ernst, M. MIRROR-CP: A Proton-Only Experiment for the Measurement of ${ }^{13} \mathrm{C}$ Spin Diffusion. Chem. Phys. Lett. 2009, 479, 296-299.

(93) Ernst, M.; Meier, M. A.; Tuherm, T.; Samoson, A.; Meier, B. H. Low-Power High-Resolution Solid-State NMR of Peptides and Proteins. J. Am. Chem. Soc. 2004, 126, 4764-4765.

(94) Bayro, M. J.; Ramachandran, R.; Caporini, M. A.; Eddy, M. T.; Griffin, R. G. Radio Frequency-Driven Recoupling at High Magic-Angle Spinning Frequencies: Homonuclear Recoupling sans Heteronuclear Decoupling. J. Chem. Phys. 2008, 128, 11.

(95) Ye, Y. Q.; Malon, M.; Martineau, C.; Taulelle, F.; Nishiyama, Y. Rapid Measurement of Multidimensional ${ }^{1} \mathrm{H}$ Solid-State NMR Spectra at Ultra-Fast MAS Frequencies. J. Magn. Reson. 2014, 239, 75-80. 